\title{
Lexis
}

Journal in English Lexicology

11 | 2018

Lexis in Languages for Specific Purposes (LSP)

\section{Étude lexicométrique des termes centraux dans un corpus d'articles scientifiques en orthophonie}

Frédérique Brin-Henry, Évelyne Jacquey et Sandrine Ollinger

\section{(2) OpenEdition}

Journals

Édition électronique

URL : http://journals.openedition.org/lexis/1201

DOI : $10.4000 /$ lexis. 1201

ISSN : 1951-6215

Éditeur

Université Jean Moulin - Lyon 3

Référence électronique

Frédérique Brin-Henry, Évelyne Jacquey et Sandrine Ollinger, «Étude lexicométrique des termes centraux dans un corpus d'articles scientifiques en orthophonie », Lexis [En ligne], 11 | 2018, mis en ligne le 30 avril 2018, consulté le 01 mai 2019. URL : http://journals.openedition.org/lexis/1201 ; DOI : $10.4000 /$ lexis. 1201

Ce document a été généré automatiquement le 1 mai 2019.

\section{(i) (9)}

Lexis is licensed under a Creative Commons Attribution-NonCommercial-NoDerivatives 4.0 International License. 


\title{
Étude lexicométrique des termes centraux dans un corpus d'articles scientifiques en orthophonie
}

\author{
Frédérique Brin-Henry, Évelyne Jacquey et Sandrine Ollinger
}

Les auteures sont membres de l'Équipe Lexique du laboratoire ATILF UMR 7118 CNRS/Université de Lorraine. Elles remercient vivement les orthophonistes ayant accepté de participer bénévolement à la phase d'expertise de l'étude, ainsi que Jessika Pérignon pour son précieux concours dans la constitution du corpus.

\section{Introduction}

1 Les résultats de la première expérience lexicographique en orthophonie sont parus en 1997 avec la première édition du Dictionnaire d'orthophonie [Brin et al.]. Ce travail disciplinaire a été enrichi, amendé au cours des deux éditions qui se sont succédé en 2004 et 2011, la quatrième édition devant paraître en 2018. Cet ouvrage, élaboré de façon relativement intuitive, quoique conséquent (plus de 3.000 entrées et définitions), révèle la multiplicité des domaines scientifiques contribuant à la discipline, ainsi que la complexité des réseaux lexicaux en jeu.

2 Afin de poursuivre ce travail d'examen de la terminologie orthophonique, nous avons adopté un point de vue sémantico-syntaxique (Brin-Henry [2011], Brin-Henry \& Knittel [2015], [2016]), mais également discursif (Brin-Henry [2014], Brin-Henry \& Knittel [2016]) grâce à la recherche de routines et motifs discursifs (Née, Sitri \& Veiniard [2014]). Pour ce faire nous avons constitué un premier corpus de comptes rendus de bilan orthophonique (Brin-Henry [2011]), puis un second corpus (constitué d'articles de revue scientifique) dans le cadre du projet OrthoCorpus ${ }^{1}$ (2015-2017).

Notre objectif à long terme est de constituer une représentation conceptuelle novatrice des pathologies du langage et de la communication, plus argumentée qu'une simple expertise intuitive, mais qui s'éloigne des considérations biomédicales. Prenant en compte ce que disent les orthophonistes, et la façon dont ils l'expriment, notamment au 
moyen de ces analyses terminologiques, discursives et sémantico-syntaxiques, nous cherchons à faire émerger des propriétés et des arguments spécifiques au domaine de l'orthophonie, qui constitueront les fondements de cette représentation conceptuelle.

4 Par le biais de cet objectif, nous abordons l'étude des relations entre la terminologie orthophonique, les représentations des professionnels et la réalité de la situation des patients et de leur entourage. Au fur et à mesure de nos explorations, nous avons engagé une réflexion sur le statut terminologique et la puissance d'une unité lexicale (UL) au sein d'un discours de spécialité (L'Homme [2005], Charaudeau \& Maingueneau [2002]). Ainsi nous établissons plusieurs degrés d'importance pour les UL du discours des orthophonistes, les UL se situant sur un continuum allant du plus général («mardi», « adulte », « domicile », « entreprendre », etc.) au plus spécifique (« dysphasie », « trouble de l'articulation ", "rééducation", etc.). Ces explorations nous semblent primordiales pour montrer que le discours et la terminologie orthophoniques sont constitués de syntagmes particuliers, distincts de ceux des autres professionnels de santé et de la langue générale (Cabré [2012]).

5 Nous avons en particulier considéré des UL que nous appelons «centrales » et qui font l'objet de cet article. Nous interrogeons ici les relations de congruence entre une intuition experte, des critères de fréquence ou le comportement remarquable d'une unité lexicale, l'exploration semi-automatique des dépendances de cette UL, et un calcul de spécificité opéré par l'examen de segments répétés. Le présent travail s'intéresse à l'établissement d'une méthode reproductible portant sur des unités lexicales centrales pour le domaine. Il s'agit d'UL appartenant indiscutablement à la langue des orthophonistes et qui possèdent un sens spécifique et important pour la discipline. Ces termes sont selon nous utilisés de façon centrale, mais pas forcément fréquente. Cet article présente les étapes de sélection de cette vingtaine d'UL constituant une liste de termes centraux. La méthodologie employée, que nous allons d'abord détailler dans les sections suivantes, comprend plusieurs étapes successives portant sur les UL.

\section{Première étape : Sélection et validation par les experts}

6 Cette première étape a permis de constituer une liste et de la soumettre à un panel d'experts pour valider l'intuition initiale de sélection des UL centrales. Nous détaillons cidessous les étapes proposées.

\subsection{Sélection initiale}

7 À partir d'une intuition experte, une liste de 21 unités lexicales (UL) a été établie par une des auteures, elle-même orthophoniste, qui s'est prononcée sur l'intérêt de chaque terme. Cette première liste a été modifiée, notamment par l'ajout d'autres termes extraits d'une relecture du Dictionnaire d'orthophonie.

8 La liste finalisée est la suivante : « articulation, assourdissement, attaque, atteinte, bilan, cérébral, compréhension, correction, déviant, diagnostic, domaine, expression, jargon, mode, origine, problème, production, réalisation, structure, transformations, transposition ». Elle comprend des formes fléchies qui semblaient $a$ priori plus fréquentes 
(«transformations »), deux adjectifs (« cérébral», « déviant»), mais elle est principalement constituée de noms singuliers.

\subsection{Enquête experte à partir de critères sélectionnés}

9 Nous souhaitions recueillir les représentations d'experts du domaine de l'orthophonie concernant ces UL, et également valider la sélection initiale. Cette liste a donc été proposée à 31 experts-orthophonistes afin de vérifier la présence de ce caractère central, établie à partir des propriétés suivantes : polysémie, usage, occurrences révélatrices des pratiques, et un ou plusieurs sens spécifiques au domaine. L'objectif était d'obtenir un avis concernant l'usage spécifique de l'UL dans la pratique de l'orthophonie et son importance (voir questions 2 et 3, en annexe), mais également un avis concernant les formes généralement usitées pour chacune des UL (voir questions 4 et 5 , en annexe). Enfin le critère de polysémie, bien que non consensuel sur le plan théorique dans ses relations à la terminologie, nous a paru intéressant à faire évaluer par les experts de façon intuitive (voir question 1, en annexe) pour identifier la réalisation de ce trait dans le discours (Lino [2006]).

Le questionnaire a été envoyé par courriel à 31 orthophonistes, tous pratiquant l'orthophonie, et pour certains d'entre eux assumant un autre rôle complémentaire. Cet échantillon de professionnels a été constitué à partir de plusieurs critères : l'expérience $(+$ ou - de cinq ans d'exercice), l'usage des écrits scientifiques (publiant ou lecteur), le type d'exercice et enfin la démarche réflexive par rapport à la profession dans sa globalité (praticien, enseignant, responsable dans la formation initiale, responsable syndical).

11 Les orthophonistes étaient invités (de juillet à septembre 2017) à renseigner un tableur Excel dont les cellules pouvaient être remplies grâce à un menu déroulant (présenté en annexe). Une colonne permettait d'ajouter des remarques. La consigne fournie était de proposer une réponse relativement spontanée. Certains orthophonistes ont demandé plus de précisions quant aux enjeux du questionnement et l'utilisation des réponses.

Nous avons obtenu les réponses de 11 experts. La répartition de ces professionnels est la suivante, présentée selon les critères (non exclusifs²) et le fait qu'ils aient participé ou non à cette enquête.

Tableau 1 : Profils des experts ayant répondu au questionnaire

\begin{tabular}{|l|l|l|}
\hline Répartition des experts & $\begin{array}{l}\text { Total des } \mathbf{3 1} \\
\text { orthophonistes }\end{array}$ & $\begin{array}{l}\text { Sous-total des 11 } \\
\text { répondants }\end{array}$ \\
\hline Expérience : & & \\
\hline Expérimentés/non expérimentés & $26 / 5$ & $9 / 2$ \\
\hline Usage des écrits : & & \\
\hline Lecteurs/publiants & $19 / 12$ & $6 / 5$ \\
\hline Type d'exercice : & & \\
\hline Chercheurs/enseignants/praticiens & $6 / 7 / 11$ & $3 / 2 / 4$ \\
\hline
\end{tabular}




\begin{tabular}{|l|l|l|}
\hline Démarche professionnelle : & & \\
\hline $\begin{array}{l}\text { Responsables formation initiale/ } \\
\text { syndicaux }\end{array}$ & $3 / 4$ & $1 / 1$ \\
\hline
\end{tabular}
retrouvent que des praticiens et des lecteurs. examinées. L'ensemble des résultats a été parcouru afin de mesurer l'amplitude des divergences entre les experts. Puis nous avons établi des résultats moyens (sélectionnés par le plus grand nombre d'experts), et procédé au calcul d'une médiane pour les résultats chiffrés. Enfin, nous avons comparé avec les cotations proposées intuitivement par l'auteure orthophoniste afin de mesurer la convergence entre son intuition et celle du panel d'experts ainsi constitué.

\subsection{Résultats au questionnaire}

15 Nous présentons tout d'abord les résultats en fonction des réponses majoritaires ou des médianes.

Tableau 2 : Moyenne des réponses obtenues au questionnaire proposé aux orthophonistes-experts

\begin{tabular}{|c|c|c|c|c|c|}
\hline $\begin{array}{l}\text { UL } \\
\text { sélectionnée }\end{array}$ & $\begin{array}{l}\text { possède } \\
\text { plusieurs sens } \\
\text { en } \\
\text { orthophonie } \\
\text { selon le } \\
\text { contexte? } \\
\text { (ex.: } \\
\text { référence } \\
\text { anatomique et } \\
\text { référence } \\
\text { linguistique) }\end{array}$ & $\begin{array}{l}\text { spécifique aux } \\
\text { orthophonistes } \\
\text { par rapport à } \\
\text { d'autres } \\
\text { professions de } \\
\text { santé? De pas } \\
d \mathfrak{l} \text { tout (0) à } \\
\text { très spécifique } \\
\text { (5) }\end{array}$ & $\begin{array}{l}\text { est important } \\
\text { dans les écrits, } \\
\text { car révélateur de } \\
\text { la pratique des } \\
\text { orthophonistes? }\end{array}$ & $\begin{array}{l}\text { plutôt } \\
\text { employé } \\
\text { au } \\
\text { singulier, } \\
\text { au pluriel } \\
\text { ou les } \\
\text { deux? }\end{array}$ & $\begin{array}{l}\text { peut être } \\
\text { employé } \\
\text { seul? (Sinon, } \\
\text { il nécessite } \\
\text { toujours un } \\
\text { complément) }\end{array}$ \\
\hline articulation & oui & 3 & Oui & $\begin{array}{l}\text { plutôt } \\
\text { sing }\end{array}$ & oui \\
\hline assourdissement & non & 4 & Oui & $\begin{array}{l}\text { plutôt } \\
\text { sing }\end{array}$ & oui \\
\hline attaque & oui & 2 & Oui & $\begin{array}{l}\text { plutôt } \\
\text { sing }\end{array}$ & oui \\
\hline atteinte & oui & 0 & Oui & les 2 & non \\
\hline bilan & non & 2 & Oui & $\begin{array}{l}\text { plutôt } \\
\text { sing }\end{array}$ & oui \\
\hline cérébral & non & 0 & Oui & les 2 & non \\
\hline
\end{tabular}




\begin{tabular}{|c|c|c|c|c|c|}
\hline compréhension & oui & 3 & Oui & $\begin{array}{l}\text { plutôt } \\
\text { sing }\end{array}$ & oui \\
\hline correction & oui & 0 & Non & $\begin{array}{l}\text { plutôt } \\
\text { sing }\end{array}$ & non \\
\hline déviant & non & 1 & Oui & les 2 & oui \\
\hline diagnostic & non & 1 & Oui & $\begin{array}{l}\text { plutôt } \\
\text { sing }\end{array}$ & oui \\
\hline domaine & non & 0 & Oui & les 2 & non \\
\hline expression & oui & 4 & Oui & $\begin{array}{l}\text { plutôt } \\
\text { sing }\end{array}$ & oui \\
\hline jargon & oui & 4 & Oui & $\begin{array}{l}\text { plutôt } \\
\text { sing }\end{array}$ & oui \\
\hline mode & oui & 1 & Oui & $\begin{array}{l}\text { plutôt } \\
\text { sing }\end{array}$ & non \\
\hline origine & non & 0 & Non & égalité & non \\
\hline problème & oui & 0 & Non & les 2 & oui \\
\hline production & non & 3 & Oui & les 2 & oui \\
\hline réalisation & égalité & 1 & Oui & $\begin{array}{l}\text { plutôt } \\
\text { sing }\end{array}$ & non \\
\hline structure & oui & 1 & Oui & les 2 & non \\
\hline transformations & oui & 2 & Oui & $\begin{array}{l}\text { plutôt } \\
\text { pluriel }\end{array}$ & oui \\
\hline transposition & oui & 3 & Oui & les 2 & oui \\
\hline
\end{tabular}

Bien que certains experts n'aient pas pu répondre à quelques questions, nous avons considéré qu'un résultat supérieur ou égal à la majorité absolue (6) représentait un seuil acceptable pour la prise en compte des réponses.

On constate que la plupart des termes sont considérés comme importants (sauf " correction », « origine ", " problème »), et comme étant plutôt employés au singulier et plutôt sans dépendance. Pour ce qui concerne la polysémie et la spécificité de l'usage par les orthophonistes (comparé à l'usage par d'autres professionnels de santé), les avis semblent beaucoup plus partagés. La sous-section suivante détaille ces résultats en fonction de chaque critère.

18 Au moyen de la création d'un tableau croisé dynamique, les résultats ainsi regroupés permettent d'obtenir une présentation de chaque critère interrogé. Pour plus de clarté, nous limitons la présentation des résultats aux UL ayant un « score » de 6 et plus. 


\subsubsection{Caractère polysémique}

19 Le caractère polysémique est distribué de façon relativement large dans les réponses obtenues, rendant difficile une conclusion autre que sur les extrêmes. Le tableau suivant classe les résultats du plus polysémique au moins polysémique selon les professionnels interrogés.

Tableau 3 : Unités linguistiques perçues comme polysémiques par les experts (en nombre de réponses)

\begin{tabular}{|l|l|}
\hline UL & Nb \\
\hline articulation & 9 \\
\hline atteinte & 9 \\
\hline expression & 9 \\
\hline problème & 9 \\
\hline structure & 9 \\
\hline attaque & 7 \\
\hline mode & 7 \\
\hline transformations & 7 \\
\hline compréhension & 6 \\
\hline correction & 6 \\
\hline jargon & 6 \\
\hline
\end{tabular}

Les termes ressentis comme étant les moins polysémiques sont " cérébral », « diagnostic » et « assourdissement ».

\subsubsection{Caractère spécifique du terme en orthophonie par comparaison à d'autres professions de santé}

21 Ce critère a été exploré en demandant aux experts s'ils considéraient ce terme comme étant spécifique aux orthophonistes par rapport à son usage par d'autres professionnels de santé. Nous avons considéré que les cotations $3,4,5$, accordent au terme un caractère spécifique, et que les réponses $0,1,2$ montraient que les répondants n'accordaient pas ce caractère à l'UL en question.

Tableau 4 : Unités linguistiques perçues comme spécifiques par les experts (en nombre de réponses)

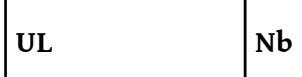




\begin{tabular}{|l|l|}
\hline jargon & 11 \\
\hline assourdissement & 10 \\
\hline expression & 9 \\
\hline production & 9 \\
\hline articulation & 7 \\
\hline compréhension & 7 \\
\hline transposition & 6 \\
\hline
\end{tabular}

Il s'avère à l'examen des réponses que la question posée manquait de clarté. Nous aurions dû formuler cette question en demandant si le terme en question, dans le discours orthophonique, avait des usages différents de ceux envisagés dans les discours émanant d'autres professionnels de santé que les orthophonistes. En réduisant le critère aux usages, les experts pourraient se prononcer plus efficacement. Il en ressort néanmoins que, selon la majorité des experts, seuls sept noms parmi les 21 proposés possèdent cette caractéristique. "Jargon» et "assourdissement" sont quasi unanimement ressentis comme spécifiques au discours des orthophonistes.

\subsubsection{Degré d'importance dans les écrits (car révélateurs des pratiques)}

La question posée semble plus explicite pour les orthophonistes et plus proche de la notion de centralité. Ainsi les réponses obtenues sont plus homogènes, et la plupart des termes $(18 / 21)$ sont considérés comme importants et révélateurs des pratiques.

Tableau 5 : Unités linguistiques perçues comme importantes par les experts (en nombre de réponses)

\begin{tabular}{|l|l|}
\hline UL & Nb \\
\hline articulation & 11 \\
\hline bilan & 11 \\
\hline compréhension & 11 \\
\hline diagnostic & 11 \\
\hline expression & 11 \\
\hline production & 11 \\
\hline assourdissement & 10 \\
\hline jargon & 10 \\
\hline attaque & 8 \\
\hline
\end{tabular}




\begin{tabular}{|l|l|}
\hline déviant & 8 \\
\hline transformations & 8 \\
\hline transposition & 8 \\
\hline structure & 7 \\
\hline atteinte & 6 \\
\hline cérébral & 6 \\
\hline domaine & 6 \\
\hline mode & 6 \\
\hline réalisation & 6 \\
\hline
\end{tabular}

24 Seuls « origine ", « correction » et « problème » ne sont pas considérés comme des termes révélateurs des pratiques des orthophonistes.

\subsubsection{Singulier/pluriel}

25 Ce paramètre a surtout été inclus dans une perspective de validation des formes proposées.

Tableau 6 : Formes pressenties par les experts comme usitées au singulier ou indifféremment au singulier/pluriel (en nombre de réponses)

\begin{tabular}{|l|l|}
\hline UL & $\mathrm{Nb}$ \\
\hline articulation & 11 \\
\hline compréhension & 11 \\
\hline jargon & 11 \\
\hline assourdissement & 10 \\
\hline diagnostic & 10 \\
\hline bilan & 9 \\
\hline expression & 9 \\
\hline attaque & 7 \\
\hline mode & 7 \\
\hline réalisation & 7 \\
\hline correction & 6 \\
\hline
\end{tabular}


\begin{tabular}{l|l} 
origine & 6
\end{tabular}

Le consensus semble être assez fort pour environ la moitié des UL. Pour huit des experts, « transformations » est sans conteste plutôt utilisé au pluriel.

Par ailleurs, les UL pressenties par plus de la moitié des experts comme pouvant être utilisées indifféremment au singulier ou au pluriel sont les suivantes: "atteinte", " domaine », « problème ", « déviant », « cérébral », « production » et « structure ».

\subsubsection{Emploi avec ou sans dépendance}

Le tableau ci-dessous présente les réponses majoritairement obtenues à la question de l'usage de l'UL sous une forme syntagmatique ou utilisé seul.

Tableau 7 : Formes pressenties par les experts comme usitées sans dépendance (en nombre de réponses)

\begin{tabular}{|l|l|}
\hline UL & Nb \\
\hline articulation & 9 \\
\hline jargon & 9 \\
\hline assourdissement & 8 \\
\hline attaque & 8 \\
\hline expression & 7 \\
\hline production & 7 \\
\hline transformations & 7 \\
\hline bilan & 6 \\
\hline compréhension & 6 \\
\hline déviant & 6 \\
\hline diagnostic & 6 \\
\hline problème & 6 \\
\hline
\end{tabular}

Ce caractère ne permet pas de distinguer les termes centraux des autres. En effet ce caractère semble davantage lié aux propriétés linguistiques du terme lui-même.

Cependant nous pensons qu'il peut également s'agir, dans ces usages préférentiels sans dépendance, d'une forme elliptique. C'est notamment le cas de "bilan ", qui sous-entend très fréquemment qu'il s'agit de « bilan orthophonique ». 


\subsubsection{Ajustement de l'intuition initiale au regard des réponses obtenues} grisée l'estimation initiale de l'auteure experte en orthophonie. En abscisse figure la
cotation attribuée à l'UL, en ordonnée le nombre de réponses obtenues pour chaque
cotation. La figure 1 présente le cas de « cérébral ».

Figure 1 : Distribution des réponses obtenues concernant la spécificité de « cérébral »

\begin{tabular}{|c|c|c|c|c|c|c|c|}
\hline & & & & cérébral & & & \\
\hline & 6 & $x$ & & & & & \\
\hline & 5 & & & & & & \\
\hline \multirow[t]{7}{*}{$\mathrm{nb}$} & 4 & & & & & & \\
\hline & 3 & & & & & & \\
\hline & 2 & & $\mathrm{x}$ & $\mathrm{x}$ & & & \\
\hline & 1 & & & & & $x$ & \\
\hline & 0 & & & & & & \\
\hline & & 0 & 1 & 2 & 3 & 4 & 5 \\
\hline & & & cotation & & & & \\
\hline
\end{tabular}

Il en ressort un accord entre tous les professionnels, une proximité forte pour quelques UL ( « cérébral », « correction », « jargon », « origine », « problème », " production »), une proximité acceptable pour certaines UL ( " attaque », " atteinte ", " compréhension », « domaine », " expression », "structure »), et moins marquée pour d'autres («bilan », « mode », « réalisation »).

Pour les quatre UL « déviant ", « diagnostic », " transformations », " transposition », les avis divergent et le plus souvent la distribution des réponses est très large, comme le montre la figure ci-dessous.

Figure 2 : Distribution des réponses concernant la spécificité de « transposition »

\begin{tabular}{|c|c|c|c|c|c|c|c|}
\hline & 7 & & & & & & \\
\hline & 6 & & & transposition & & & \\
\hline & 5 & & & & & & \\
\hline \multirow[t]{6}{*}{$\mathrm{nb}$} & 4 & & & & & & \\
\hline & 3 & & & & & $x$ & \\
\hline & 2 & & $x$ & & $x$ & & \\
\hline & 1 & $x$ & & $x$ & & & $\mathrm{x}$ \\
\hline & 0 & & & & & & \\
\hline & & 0 & 1 & 2 & 3 & 4 & 5 \\
\hline
\end{tabular}

Cette comparaison nous permet de dire qu'une large majorité des UL sélectionnées au départ (16/21) semblent dignes d'intérêt, et que l'intuition initiale de l'expert-auteur est cohérente avec l'avis des orthophonistes interrogés. Pour les quatre UL restantes, les experts n'atteignent pas de consensus clair sur l'importance du terme; nous pensons que ce phénomène est causé par des acceptions moins bien définies des termes en question, 
émanant de points de vue théoriques divers. Par exemple la notion de "déviance » est actuellement remise en cause dans les définitions des troubles développementaux du langage et cela a pu influencer les experts.

\subsubsection{Ajustement par l'examen des corrélations}

Nous présentons ici les résultats en cumulant certaines propriétés supposées, afin d'en déterminer les plus critiques pour établir le caractère central de l'UL. En d'autres termes, est-ce qu'une UL considérée comme spécifique (cotation à $3,4,5$ ), importante et révélatrice des pratiques par les experts, correspond à ce qu'on entendrait par «terme central»?

Le tableau 8 suivant montre les neuf UL résultants de la combinaison de ces propriétés. La prise en compte du critère selon lequel le terme est perçu comme étant principalement usité sans dépendance n'amène pas de modification de la liste. On pourrait considérer qu'il s'agit ici de la liste des termes centraux.

Tableau 8 : Liste des UL considérées comme importantes, spécifiques, polysémiques et sans dépendance par les experts (en nombre de réponses)

\begin{tabular}{|l|l|}
\hline UL & Nb \\
\hline articulation & 5 \\
\hline expression & 5 \\
\hline compréhension & 3 \\
\hline jargon & 3 \\
\hline production & 3 \\
\hline assourdissement & 1 \\
\hline bilan & 1 \\
\hline transformations & 1 \\
\hline transposition & 1 \\
\hline
\end{tabular}

Cependant, aucun de ces termes n'obtient la majorité des réponses obtenues, ce qui va à l'encontre du parti pris initial. Si l'on enlève le caractère de ne pas avoir de dépendance, le terme « expression » apparait alors comme significativement pertinent (7 réponses).

Si l'on omet le caractère polysémique, la liste est alors réduite et dans ce cas, «bilan » et «transformations » disparaissent. Huit experts considèrent « assourdissement » comme porteur des trois propriétés considérées (voir tableau 9). 
Tableau 9 : Liste des UL considérées comme importantes, spécifiques et sans dépendance par les experts (en nombre de réponses)

\begin{tabular}{|l|l|}
\hline Étiquettes de lignes & $\mathbf{N b}$ \\
\hline assourdissement & 8 \\
\hline jargon & 8 \\
\hline articulation & 6 \\
\hline expression & 6 \\
\hline production & 6 \\
\hline
\end{tabular}

37 Le tableau 10 ci-dessous présente les résultats obtenus en ne tenant compte que des critères de l'importance pressentie ainsi que la spécificité du terme selon les experts.

Tableau 10 : Liste des UL considérées comme importantes et spécifiques par les experts (en nombre de réponses)

\begin{tabular}{|l|l|}
\hline Étiquettes de lignes & $\mathbf{N b}$ \\
\hline assourdissement & 10 \\
\hline jargon & 10 \\
\hline expression & 9 \\
\hline production & 9 \\
\hline articulation & 7 \\
\hline compréhension & 7 \\
\hline transposition & 6 \\
\hline
\end{tabular}

En réduisant le nombre de critères, on obtient une liste un peu enrichie par rapport à la précédente, consensuelle, mais sans réelle modification des termes principaux.

Le tableau 11 explore l'intérêt de considérer des termes pressentis comme importants, mais utilisés principalement sans dépendance, afin de voir si le critère syntaxique ou elliptique de l'UL pourrait être primordial.

Tableau 11 : Liste des UL considérées comme importantes et sans dépendance par les experts (en nombre de réponses)

\begin{tabular}{|l|l|}
\hline Étiquettes de lignes & $\mathrm{Nb}$ \\
\hline articulation & 9 \\
\hline
\end{tabular}




\begin{tabular}{|l|l|}
\hline assourdissement & 8 \\
\hline jargon & 8 \\
\hline attaque & 7 \\
\hline expression & 7 \\
\hline production & 7 \\
\hline bilan & 6 \\
\hline compréhension & 6 \\
\hline diagnostic & 6 \\
\hline
\end{tabular}

40 Y réapparaissent alors les termes de «diagnostic » et de «bilan ", particulièrement signifiants dans la pratique des orthophonistes, qui sont effectivement fréquemment utilisés seuls mais peuvent également comporter de nombreuses dépendances comme le montrera la section 2.3.

41 Enfin, si l'on considère l'application de filtres ne sélectionnant qu'un seul critère, on perd en précision. En effet le critère unique de l'importance dans les écrits n'est pas très discriminant parce que quasiment toutes les UL portent cette caractéristique, à l'exception des UL « origine » et "problème ». De même, le critère unique de "spécifique aux orthophonistes par rapport à d'autres professionnels de santé » ne permet pas de trouver de nouveaux arguments en faveur d'une définition du caractère central des UL de la liste proposée.

Cette étape de validation experte a permis de dégager plusieurs éléments essentiels à la constitution d'une définition stable de «terme central». Ainsi, le critère de polysémie étant peu discriminant, il semble que les UL centrales aient été celles considérées comme importantes dans les écrits (révélatrices des pratiques), et spécifiques (usages particuliers de cette UL par les orthophonistes en contraste avec d'autres professionnels de santé). Il reste donc a priori dans cette liste les termes suivants: "articulation", " assourdissement », «jargon», " expression», " production», avec éventuellement « bilan », « compréhension », « transposition » et « diagnostic ».

Le terme «oralité(s)» a été finalement ajouté à cette liste par son caractère particulièrement actuel, puisqu'il a constitué la thématique du dernier congrès scientifique international de la Fédération Nationale des Orthophonistes ${ }^{3}$ (Brin-Henry [2017]).

\section{Deuxième étape : analyse de corpus}

Cette étape a permis d'examiner ces UL dans le discours des orthophonistes, dans le but de déterminer les dépendances et de compléter les informations concernant les usages. Ainsi, les contextes à droite et à gauche de chaque terme, ainsi que leurs diverses variantes syntaxiques sont explorés afin de mieux établir la particularité de chaque UL. Les paragraphes suivants en détaillent les étapes. 


\subsection{Présentation du corpus et méthode d'analyse} corpus. Ainsi, procédons-nous, grâce à l'expertise disciplinaire de l'un des auteurs, à une inspection préalable des usages syntagmatiques terminologiques, fondée sur des indicateurs sémantico-syntaxiques et, dans une moindre mesure, de fréquence. L'intérêt est de montrer que les critères de richesse et de variété des dépendances syntagmatiques 
du terme et de ses emplois dans des documents principalement écrits par des orthophonistes lui donnent un caractère central.

51 La section suivante détaille cette procédure et les résultats obtenus pour l'exemple de «bilan ».

\subsubsection{L'exemple de « bilan »}

Les étapes successives opérées ont permis de sélectionner les requêtes les plus pertinentes. En premier lieu, nous avons cherché le nombre total d'occurrences de «bilan ", ainsi que ses formes fléchies dans le corpus général regroupant l'ensemble des articles. La requête initiale (Index de <"bilan. ${ }^{*} \% \mathrm{~cd}>$ avec la propriété [word] dans le corpus ORTHOCORPUS) inclut les formes commençant par les caractères BILAN, suivis par n'importe quel caractère et en majuscules et/ou minuscules.

53 Huit items ont été obtenus pour 1675 occurrences distribuées comme suit : 1637 «bilan (s) » (toutes combinaisons de majuscules/minuscules combinées), 1 «bilan-pathologie » et 1 «bilanter». Lorsque l'on poursuit la recherche dans le sous-corpus regroupant les articles rédigés par au moins un orthophoniste, le nombre d'occurrences reste élevé avec 1315 items. Pour ce qui est du sous-corpus d'articles ayant été rédigés par d'autres professionnels, on retrouve seulement 250 occurrences de «bilan». A priori, les orthophonistes semblent donc être des utilisateurs privilégiés de ce terme. «Bilanter»a d'ailleurs été utilisé par une orthophoniste. Un étiquetage manuel a permis de caractériser les classes syntaxiques dans les contextes à droite de « bilan ».

Tableau 12 : Classes syntaxiques des contextes à droite de «bilan» (en nombre d'occurrences)

\begin{tabular}{|l|l|l|}
\hline Dépendances de bilan (à droite) & Nb & Exemple \\
\hline adj & 592 & bilan orthophonique \\
\hline$\varnothing$ & 380 & \\
\hline V & 153 & bilan comprend \\
\hline de+dét+N & 130 & bilan de la déglutition \\
\hline de+N & 109 & bilan de surdité \\
\hline ppassé & 35 & bilan effectué \\
\hline prép & 16 & bilan chez l'adulte \\
\hline Nom propre & 14 & bilan ERLA \\
\hline pprésent & 10 & bilan comportant \\
\hline de+num & 8 & bilan des 4 ans \\
\hline N & 5 & bilan ORL \\
\hline de+Nom propre & 3 & bilan de F. Coquet \\
\hline
\end{tabular}




\begin{tabular}{|l|l|l|}
\hline prép+N & 1 & bilan sur support \\
\hline Total & 1456 & \\
\hline
\end{tabular}

La plupart des occurrences de «bilan » sont suivies d'adjectifs taxinomiques permettant de situer le type de bilan, que ce soit pour en caractériser le contenu et l'objet : « logicomathématiques", «neuromoteur", «langagier ", «visuel », «psychomoteur ", "psycholinguistique », ou bien pour spécifier la profession concernée : «logopédique », " orthophonique", «neuropsychologique», «médical», "psychologique», « endocrinologique». "Bilan orthophonique» est de loin le plus fréquent (177 occurrences).

Le tableau suivant détaille les thématiques et les champs sémantiques qui transparaissent dans les concordances.

Tableau 13 : Fréquence des champs sémantiques des contextes à droite et à gauche de «bilan » (en nombre d'occurrences)

\begin{tabular}{|c|c|c|c|}
\hline & Dépendances de bilan (à droite) & $\mathrm{Nb}$ & Exemple \\
\hline \multirow{5}{*}{ Type de bilan } & règlementation & 286 & bilan de dépistage \\
\hline & modalité & 130 & bilan clinique \\
\hline & qualificatif & 67 & bilan approfondi \\
\hline & modalité de passation & 20 & bilan informatisé \\
\hline & nom de bilan & 16 & bilan ERLA \\
\hline Évaluateur & autre profession & 58 & bilan psychologique \\
\hline \multirow{3}{*}{ Domaine } & mécanisme exploré & 255 & bilan de langage oral \\
\hline & pathologie concernée & 47 & bilan de la dysarthrie \\
\hline & pathologie (personne) & 30 & bilan de l'adolescent \\
\hline \multirow{3}{*}{ Autre } & autre & 4 & \\
\hline & $\varnothing$ & 543 & \\
\hline & Total & 1456 & \\
\hline
\end{tabular}

Les adjectifs qualificatifs utilisés en contexte à droite de «bilan» montrent des conceptions soit temporelles (" préopératoire »), soit mesurées du bilan (« approfondi »). De plus, le patient est mentionné dans quelques cas pour caractériser le type du bilan (« bilan de l'enfant », « chez l'adulte »). Dans environ un tiers des cas, aucune dépendance n'est retrouvée, «bilan » est alors utilisé seul. L'aspect réglementaire permet également de caractériser le type de bilan proposé, et enfin le nom du bilan apparaît également dans 
ces occurrences. Les verbes utilisés montrent que le bilan est perçu comme comportant des items ( repose», "nécessite»), mais également comme un moyen d'évaluation (« déterminer », « devoir », « être », « évalue »).

La même procédure est adoptée concernant les contextes à gauche. On y retrouve moins d'occurrences, situant principalement le bilan dans le temps («lors du», « au cours du», " après ») et localisant les activités dans la phase de bilan ("dans le cadre de », " dans le»). De plus, des adjectifs tels que "premier », "nouveau », permettent de qualifier ce bilan également dans une dimension temporelle. Les phases du bilan sont précisées, on retrouve notamment mention de la "réalisation", le "résultat ", la "rédaction » du bilan. Sur le plan des définitions existantes, dans le Dictionnaire d'orthophonie, l'entrée est proposée à "bilan orthophonique», mais "bilan» n'y figure pas seul. "Bilan » est considéré comme étant polysémique par le dictionnaire Larousse.

Pour conclure concernant "bilan ", l'analyse des concordances montre qu'il s'agit d'un terme employé de façon fréquente, surtout chez les orthophonistes, qui évoque une activité professionnelle spécifique, avec des variantes concernant les domaines et le type de bilan. Le processus de passation est relativement détaillé. De plus, on remarque par contraste la présence fréquente d'autres types de bilan que le «bilan orthophonique ». Cette UL adopte de nombreuses dépendances disciplinaires taxinomiques. Enfin la création d'un néologisme sous la forme du verbe «bilanter » est à noter. Ce processus de dénominalisation semble intéressant pour montrer l'intégration spécifique du processus de bilan dans le discours, au moyen d'une forme verbale.

Ce terme est donc considéré comme central au vu de l'analyse de ses concordances dans le sous-corpus d'articles rédigés par au moins un professionnel orthophoniste et dans le sous-corpus d'articles rédigés par d'autres professionnels.

\subsubsection{Conclusion intermédiaire}

Les deux premières sections de cet article ont décrit les étapes d'examen des UL selon deux procédures complémentaires : une validation par des experts orthophonistes, et un examen des contextes à droite et à gauche des UL apparaissant dans un corpus d'articles scientifiques spécialisés. Un panel d'experts a été sollicité pour valider une liste initiale de 21 UL pouvant être considérées comme centrales. Nous avons présenté dans cette deuxième section une étape d'examen manuel spécialisé des contextes dans lesquels apparaissent les 21 UL sélectionnées.

Le tableau 14 ci-dessous répertorie la liste initiale, ainsi que les UL considérées comme centrales selon les deux premières étapes. Le point d'interrogation qui suit l'UL signale un doute quant à son caractère central, qui donc réclame une étape supplémentaire approfondissant les analyses. Cela est le cas lorsque les avis étaient trop divergents chez les experts, ou bien lorsque les indicateurs ne permettaient pas clairement d'établir le caractère central de l'UL dans l'étape d'analyse des concordances. 
Tableau 14 : Listes des UL centrales examinées selon les trois étapes d'opinion experte, d'analyse des concordances et de l'analyse des segments répétés

\begin{tabular}{|c|c|c|c|}
\hline $\begin{array}{l}\text { Liste initiale des } \\
\text { UL centrales }\end{array}$ & $\begin{array}{l}\text { UL centrales selon les } \\
\text { experts (à confirmer } \\
\text { si suivi de ?) }\end{array}$ & $\begin{array}{l}\text { UL centrales selon } \\
\text { l'analyse manuelle des } \\
\text { concordances (à confirmer } \\
\text { si suivi de?) }\end{array}$ & 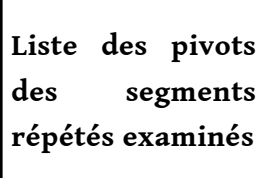 \\
\hline articulation & articulation & articulation & articulation \\
\hline assourdissement & assourdissement & assourdissement & \\
\hline attaque & & attaque & \\
\hline \multicolumn{4}{|l|}{ atteinte } \\
\hline bilan & bilan? & bilan & bilan \\
\hline \multicolumn{4}{|l|}{ cérébral } \\
\hline compréhension & compréhension? & & \\
\hline correction & & correction? & \\
\hline \multicolumn{4}{|l|}{ déviant } \\
\hline diagnostic & diagnostic? & diagnostic & diagnostic \\
\hline domaine & & domaine & domaine? \\
\hline expression & expression & & expression? \\
\hline jargon & jargon & jargon & jargon \\
\hline mode & & mode & \\
\hline \multicolumn{4}{|l|}{ origine } \\
\hline problème & & & problème? \\
\hline production & production & & \\
\hline réalisation & & réalisation & \\
\hline structure & & structure? & \\
\hline transformations & & transformations? & \\
\hline \multirow[t]{2}{*}{ transposition } & transposition? & transposition? & \\
\hline & & oralité & oralité \\
\hline
\end{tabular}


61 Nous avons souhaité enrichir nos observations en procédant à l'examen complémentaire de la façon dont ces UL apparaissent spécifiquement dans le discours des orthophonistes. Nous avons pris appui sur une étude de segments répétés dans le corpus d'articles écrits par les orthophonistes, mais également en comparaison avec un corpus de langue générale. Nous avons sélectionné les UL dans la liste initiale, selon les résultats obtenus dans les deux premières étapes d'analyse (voir tableau 14). Nous avons conservé pour la suite de l'analyse un premier groupe de cinq UL censées être centrales ("articulation », « bilan ", « diagnostic », " jargon », « oralité »), un groupe à confirmer (« expression », « domaine ») et une dernière UL censée être non centrale ( " problème »). Cette troisième étape est détaillée dans les sections suivantes.

\subsection{Examen des segments répétés}

\subsubsection{Introduction et description synthétique de la méthodologie suivie}

62 De même qu'avec l'exploration des concordances à droite et à gauche des UL analysées, notre objectif est d'étudier en quoi une analyse de leurs environnements linguistiques peut fournir des indices venant renforcer leur centralité ou au contraire l'affaiblir. L'analyse des concordances représente une première méthodologie couramment utilisée en linguistique de corpus pour étudier les usages d'une unité lexicale. La présente section la complète en appliquant une seconde méthodologie relevant de la linguistique de corpus, l'analyse des segments répétés impliquant les huit UL sélectionnées, UL que nous appellerons dorénavant des pivots.

63 Comme le présentent Mayaffre et Viprey [2012], les segments répétés peuvent être considérés comme "une spécification statistique et textuelle de la collocation ». Ils permettent de réduire la quantité et la diversité des données. Ils focalisent l'analyse sur les données qui sont récurrentes d'une part et qui apparaissent dans une fenêtre délimitée en taille. Autrement dit, la détection de segments répétés consiste à identifier des suites d'unités lexicales apparaissant plusieurs fois. Elle peut permettre de repérer aussi bien des mots composés comme " pomme de terre » ou " porte-manteau » que des constructions à verbe support comme "rédiger un article » et des associations lexicales comme celles que l'on peut trouver dans un corpus construit à l'aide de contrats d'assurance entre « assurance ", « couverture » et " risque ». Dans notre cas, l'analyse en segments répétés va venir renforcer tout ou partie de ce qui a été découvert grâce à l'analyse en concordances.

Pour réaliser cette étude, les segments répétés autour des huit pivots sont recherchés dans le sous-corpus des articles rédigés par au moins un auteur orthophoniste dans le corpus ORTHOCORPUS ${ }^{8}$. Une fois les segments répétés collectés dans le SC_ortho, ils sont recherchés dans le corpus FrWac (Baroni et al. [2009]) $)^{9}$ d'une part et, d'autre part, dans le sous-corpus des articles rédigés par des auteurs qui ne sont pas orthophonistes dans le corpus ORTHOCORPUS ${ }^{10}$. L'objectif de ces deux types de recherche est d'identifier les segments répétés qui sont surreprésentés et spécifiques du discours des orthophonistes par rapport à la langue générale (FrWac) et par rapport au discours des nonorthophonistes (SC_non-ortho). Après avoir collecté les segments répétés et mesuré leur degré de représentation, nous complétons l'analyse en classant les segments surreprésentés selon les thématiques extraites de l'analyse en concordances. Cette généralisation des segments répétés en classes peut être vue comme une forme de 
classification sémantique qui pourra fournir des informations sur les préférences sémantiques et combinatoires selon qu'un pivot est censé être central ou non, et selon que les segments dans lesquels il apparait sont surreprésentés dans le discours des orthophonistes (SC_ortho) ou dans celui des non-orthophonistes (SC_non-ortho).

À l'issue de cette première étape d'analyse, nous nous intéressons au caractère spécifique ou non des associations entre un pivot et les autres unités lexicales avec lesquelles il se combine pour former les segments répétés. Autrement dit, nous analysons la force du lien qui unit les éléments d'un segment répété. En cela, nous nous inspirons de I. Mel'cǔk et al. [1995] en étudiant dans quelle mesure les orthophonistes ont une préférence marquée pour certains cooccurrents (morphologiques, syntaxiques ou lexicaux) dans l'usage qu'ils font de chacun des huit pivots étudiés. Si une telle préférence est détectable, nous pouvons faire l'hypothèse que ce pivot possède bel et bien un sens spécifique au discours des orthophonistes (l'un des critères de l'évaluation par les experts) et qu'il sera nécessaire de décrire ce sens dans une ressource lexicographique.

\subsubsection{Extraction des segments répétés surreprésentés}

Sur le plan méthodologique, la collecte des ensembles de segments répétés a été réalisée à l'aide de la plateforme TXM. Nous empruntons à Salem [1987] le vocabulaire de description de ces segments.

67 Aucun travail particulier n'a été réalisé sur les délimiteurs de formes lors de la constitution du corpus. Il s'agit donc ici des délimiteurs par défaut de TXM. En revanche, nous avons choisi de limiter les segments répétés à l'intérieur de séquences ne comportant ni symboles ni signe de ponctuation (hors ponctuation de citation). Ainsi, le contexte "l'audition (bilan audiométrique, oto-émissions acoustiques» comporte trois séquences distinctes: "l'audition», «bilan audiométrique» et "oto-émissions acoustiques ». Aucun segment répété collecté ne chevauche plusieurs séquences ni ne contient de caractères délimiteurs de séquences.

Ce découpage en séquences réorganise les différents corpus que nous utilisons en places de tailles différentes, pouvant être occupées ou non par une occurrence des segments répétés. Afin de mener à bien nos mesures statistiques, nous avons donc calculé le nombre de k-places disponibles dans chacun des (sous-)corpus utilisés, où k correspond à l'empan, c'est-à-dire à la taille des différents segments observés. Les résultats de ces calculs sont visibles dans le tableau 15.

Tableau 15 : K-places d'ORTHOCORPUS et du FrWac

\begin{tabular}{|l|l|l|l|}
\hline & SC_ortho & SC_non-ortho & FrWac \\
\hline $\mathrm{k}=1$ & 1930271 & 1407441 & 1413243774 \\
\hline $\mathrm{k}=2$ & 1699044 & 1227546 & 1227400804 \\
\hline $\mathrm{k}=3$ & 1509335 & 1088742 & 1070925378 \\
\hline $\mathrm{k}=4$ & 1339334 & 965628 & 934975952 \\
\hline $\mathrm{k}=5$ & 1188158 & 857573 & 816339674 \\
\hline
\end{tabular}




\begin{tabular}{|l|l|l|l|}
\hline$k=6$ & 1051629 & 759895 & 711916252 \\
\hline$k=7$ & 928541 & 671736 & 620486371 \\
\hline
\end{tabular}

Comme nous pouvons le voir dans ce tableau, notre attention se concentre sur des segments de taille 1 à 7 . La taille 1 correspond aux pivots eux-mêmes, les tailles 2 à 7 des segments répétés incluent les pivots et une à six formes situées exclusivement à leur gauche ou exclusivement à leur droite. Un premier tri manuel est réalisé sur les segments collectés pour ne conserver que ceux qui rendent compte de relations syntagmatiques ou paradigmatiques impliquant les pivots. Par exemple, le segment de taille 6 « entraînant des troubles de l'articulation » n'est pas conservé. En effet, si nous pouvons y observer un lien syntagmatique entre "entraîner» et "troubles", ce lien n'implique pas " articulation ». Le lien syntagmatique entre «troubles » et " articulation», qui lui nous intéresse, se trouve collecté par l'intermédiaire d'un segment de plus petite taille.

Dès l'étape de collecte, nous constatons que nous ne pourrons pas étudier le pivot «jargon », parce qu'il n'apparaît dans aucun segment répété dans SC_ortho. Les experts orthophonistes s'accordent cependant sur la spécificité de cette UL. Nous prendrons donc garde à considérer l'étude des segments répétés comme un indice, mais qui ne suffit pas à déterminer seul le caractère central d'une UL.

Dans un premier temps, nous nous intéressons aux segments collectés en tant que tels, des unités textuelles autonomes que nous observons parce qu'elles contiennent un pivot de notre liste. Nous cherchons à déterminer si ces segments sont surreprésentés dans le discours des orthophonistes. Pour chaque segment, nous effectuons un relevé de ses fréquences absolues, dans SC_ortho d'une part et dans le corpus FrWac d'autre part. Pour rendre ces fréquences comparables, nous les normalisons à 10 millions/k-places, selon les valeurs du tableau 15.

Tableau 16 : Segments répétés surreprésentés chez les orthophonistes par rapport à la langue générale

\begin{tabular}{|l|c|c|c|c|}
\cline { 2 - 5 } \multicolumn{1}{c|}{} & $\begin{array}{l}\text { Effectif de segments sur. } \\
\text { représentés SC_ortho }\end{array}$ & $\begin{array}{l}\text { Effectif de segments sur- } \\
\text { représentés FrWac }\end{array}$ & $\begin{array}{l}\text { Effectif de segments } \\
\text { présents uniquement } \\
\text { dans SC_ortho }\end{array}$ & Total \\
\hline articulation & 64 & 0 & 26 & 90 \\
\hline bilan & 127 & 0 & 53 & 180 \\
\hline diagnostic & 63 & 0 & 47 & 110 \\
\hline domaine & 116 & 8 & 53 & 177 \\
\hline expression & 87 & 0 & 13 & 100 \\
\hline oralité & 15 & 0 & 37 & 52 \\
\hline problème & 185 & 7 & 37 & 229 \\
\hline
\end{tabular}

Cette étape nous permet d'écarter un certain nombre de segments, parce qu'ils s'avèrent surreprésentés dans la langue générale, comme "problème de santé » ou "domaines de compétences». Ces cas sont peu nombreux et concernent uniquement les pivots " domaine » et " problème », respectivement huit et sept segments écartés.

Pour d'autres segments, comme « appropriation d'une nouvelle oralité » ou « expression du ressenti de l'enfant ", nous ne tirons pas de conclusions sur leur surreprésentation dans le discours des orthophonistes, parce qu'ils sont absents du FrWac (troisième colonne du tableau 16 ci-dessus). Ces cas sont beaucoup plus nombreux. Ils représentent près d'un tiers des segments collectés pour les pivots "articulation", "bilan » et 
«domaine ». Pour les pivots «expression » et «problème », moitié moins de segments sont concernés. En revanche, la proportion augmente pour « diagnostic » et atteint plus de $70 \%$ pour « oralité ».

Nous concentrons alors notre attention sur les segments surreprésentés dans le discours des orthophonistes et présents uniquement chez les orthophonistes. Nous cherchons à déterminer s'ils sont surreprésentés dans les articles écrits exclusivement par des orthophonistes par rapport aux autres articles de la revue Réducation Orthophonique (RO). Pour chaque segment conservé, nous effectuons un relevé de ses fréquences absolues, dans SC_non-ortho. Une fois de plus, nous normalisons ses fréquences à 10 millions/kplaces, selon les valeurs du tableau 15, pour effectuer une comparaison.

De nouveaux segments sont alors écartés, comme « enjeux de l'oralité » ou " expression orale spontanée » parce qu'ils ne sont pas présents dans SC_non-ortho. Comme le montre le tableau 17 ci-dessous, un peu moins de la moitié des segments impliquant « diagnostic » ne seront pas conservés dans la suite de l'analyse, de même qu'un peu plus de la moitié des segments impliquant "domaine ", «expression » et "problème " pour aller jusqu'à près de $70 \%$ de segments non conservés pour les pivots "articulation ", «bilan» et «oralité ». Nous avons choisi de ne pas conserver les segments présents uniquement chez les orthophonistes et de nous concentrer ici sur l'usage spécifique des pivots par les orthophonistes parmi les segments utilisés en commun avec les nonorthophonistes.

Tableau 17 : Effectifs des segments répétés surreprésentés chez les orthophonistes par rapport à la revue $\mathrm{RO}$

\begin{tabular}{|l|c|c|c|c|}
\cline { 2 - 5 } \multicolumn{1}{c|}{} & $\begin{array}{l}\text { Effectif de segments sur. } \\
\text { représentés SC_ortho }\end{array}$ & $\begin{array}{l}\text { Effectif de segments sur- } \\
\text { représentés SC_non-ortho }\end{array}$ & $\begin{array}{l}\text { Effectif de segments } \\
\text { présents uniquement } \\
\text { dans SC_ortho }\end{array}$ & Total \\
\hline articulation & 24 & 6 & 60 & 90 \\
\hline bilan & 50 & 5 & 125 & 180 \\
\hline diagnostic & 39 & 24 & 47 & 110 \\
\hline domaine & 57 & 20 & 92 & 169 \\
\hline expression & 36 & 6 & 58 & 100 \\
\hline oralité & 14 & 2 & 36 & 52 \\
\hline problème & 74 & 29 & 119 & 222 \\
\hline
\end{tabular}

\subsubsection{Analyse des segments répétés surreprésentés chez les orthophonistes}

Pour aller au-delà d'une analyse strictement quantitative, nous avons classé les segments répétés sélectionnés en fonction des champs lexicaux qui ont émergé lors de l'analyse en concordances. Les étiquettes de champs lexicaux ont été homogénéisées pour éviter une trop grande disparité d'étiquettes et aider à l'émergence d'une vision plus synthétique des agencements sémantiques qui semblent être privilégiés autour des sept pivots que nous avons étudiés en détail. À l'issue de la sélection des segments répétés communs aux orthophonistes et aux non-orthophonistes, nous avons soumis 366 segments à la classification en champs lexicaux, qui se répartissent en 294 segments surreprésentés chez les orthophonistes et 72 chez les non-orthophonistes. Seuls 253 d'entre eux ont pu être classés, soit un taux moyen de classification de $69 \%$. Ce taux de classification est de $68 \%$ chez les orthophonistes (200 segments classés sur 294 ) et il est de $74 \%$ chez les nonorthophonistes (53 segments classés sur 72). Dans plusieurs cas de figure la classification n'a pas été possible. Le cas de figure le plus fréquent est l'incomplétude d'un segment pour pouvoir statuer sur une étiquette de champ lexical. C'est le cas des segments 
constitués du pivot avec une variation de la détermination uniquement («la plupart des domaines", «l'oralité », etc.), de segments introduits par des prépositions non suffisamment discriminantes ( « sur l'articulation », « de l'oralité », etc.) ou enfin le cas de segments où le pivot est le sujet d'un verbe (« bilan doit », « bilan repose », etc.).

Le tableau 18 contient la liste des étiquettes sémantiques utilisées en version étendue (colonne 1) et en version abrégée (colonne 2) ainsi que les effectifs et proportions d'utilisation de ces étiquettes pour les segments. La suite de cette section sera consacrée à une analyse plus fine des étiquettes sémantiques avec les segments répétés autour des sept pivots qui sont analysées en détail.

Tableau 18 : Liste des étiquettes de champs lexicaux utilisées pour l'analyse des segments répétés

\begin{tabular}{|c|c|c|c|c|}
\hline $\begin{array}{l}\text { Etiquettes de champs lexicaux } \\
\text { (version étendue) }\end{array}$ & $\begin{array}{c}\text { Etiquettes de champs lexicaux } \\
\text { (version abrégée) }\end{array}$ & Exemple & Effectifs & Proportions \\
\hline anatomie & anatomie & $\begin{array}{l}\text { articulations temporo- } \\
\text { mandibulaires }\end{array}$ & 6 & $2 \%$ \\
\hline ce qui est exprimé & ce qui est exprimé & expression de la pensée & 3 & $1 \%$ \\
\hline $\begin{array}{l}\text { capacité, fonction, phénomène ou } \\
\text { processus }\end{array}$ & cfpp & articulation de la parole & 34 & $9 \%$ \\
\hline $\begin{array}{l}\text { détection, qualification d'une } \\
\text { difficulté }\end{array}$ & détec_qualif (difficulté) & aborder un problème & 32 & $9 \%$ \\
\hline $\begin{array}{l}\text { détection, qualification d'une } \\
\text { pathologie }\end{array}$ & détec_qualif (patho) & bilan de dysarthrie & 10 & $3 \%$ \\
\hline $\begin{array}{l}\text { évaluation d'une capacité, } \\
\text { fonction, phénomène ou } \\
\text { processus }\end{array}$ & évaluation (cfpp) & capacités d'expression & 6 & $2 \%$ \\
\hline $\begin{array}{l}\text { évaluation négative d'une capacité, } \\
\text { fonction, phénomène ou } \\
\text { processus }\end{array}$ & évaluation négative (cfpp) & problème de compréhension & 25 & $7 \%$ \\
\hline $\begin{array}{l}\text { examen d'une capacité, fonction, } \\
\text { phénomène ou processus }\end{array}$ & examen (cfpp) & troubles de l'oralité & 7 & $2 \%$ \\
\hline démarche d'examen & examen (démarche) & diagnostic différentiel & 28 & $8 \%$ \\
\hline objectif d'examen & examen (objectif) & bilan d'évaluation & 1 & $0 \%$ \\
\hline processus d'examen & examen (processus) & Iors du bilan & 36 & $10 \%$ \\
\hline expression linguistique & expression linguistique & expression idiomatique & 4 & $1 \%$ \\
\hline importance d'une difficulté & importance (difficulté) & problème fondamental & 9 & $2 \%$ \\
\hline $\begin{array}{l}\text { modalité de réalisation d'une } \\
\text { capacité, fonction, phénomène ou } \\
\text { processus }\end{array}$ & mode de réalisation (cfpp) & mode d'articulation & 5 & $1 \%$ \\
\hline point de vue & point de ve & domaine de la morphologie & 15 & $4 \%$ \\
\hline $\begin{array}{l}\text { problème plus large que celui } \\
\text { d'une personne }\end{array}$ & problème général & problème d'évaluation & 2 & $1 \%$ \\
\hline problème mathématique & problème mathématique & résolution de problème & 8 & $2 \%$ \\
\hline professionnel pratiquant l'examen & professionnel & bilan neuropsychologique & 7 & $2 \%$ \\
\hline $\begin{array}{l}\text { stade de développement dans une } \\
\text { capacité, fonction, phénomène ou } \\
\text { processus }\end{array}$ & stade (cfpp) & oralité primaire & 7 & $2 \%$ \\
\hline temporalité & temporalité & diagnostic initial & 8 & $2 \%$ \\
\hline
\end{tabular}

78 Pour chaque pivot, nous avons rassemblé l'ensemble des champs lexicaux utilisés, recensé le nombre de segments auxquels chaque étiquette est associée et calculé le pourcentage d'utilisation de chaque étiquette par rapport au nombre total de segments classés pour ce pivot. Ce recensement est réalisé en parallèle pour les segments surreprésentés dans le discours des orthophonistes (SC_ortho) et dans celui des non-orthophonistes (SC_nonortho).

Dans le cas de «bilan », tout ou partie des champs lexicaux apparus avec les concordances (section 2.3) se retrouvent dans l'analyse des segments répétés, et cela dans des proportions comparables, excepté pour la thématique « localisation dans le temps et dans l'espace », correspondant aux thématiques « examen » (processus) et " temporalité » dans l'analyse des segments répétés. Cette disproportion pourrait être due à la focalisation sur des segments courts que l'analyse en segments répétés induit. 
Tableau 19 : Comparaison concordance et segments répétés sur « bilan »

\begin{tabular}{|c|c|c|c|c|c|}
\hline $\begin{array}{c}\text { Champs lexicaux des } \\
\text { concordances (total }=1456 \text { ) }\end{array}$ & Effectif & Pourcentage & $\begin{array}{c}\text { Champs lexicaux des segments } \\
\text { répétés classés (total }=43 \text { ) }\end{array}$ & Effectif & Pourcentage \\
\hline \multirow{2}{*}{$\begin{array}{l}\text { localisation dans le temps et } \\
\text { dans l'espace }\end{array}$} & \multirow{2}{*}{169} & \multirow{2}{*}{$12 \%$} & examen (processus) & 20 & \multirow{2}{*}{$53 \%$} \\
\hline & & & temporalité & 3 & \\
\hline type de bilan / modalité & 130 & \multirow{3}{*}{$15 \%$} & \multirow{3}{*}{ examen (démarche) } & \multirow{3}{*}{8} & \multirow{3}{*}{$19 \%$} \\
\hline type de bilan / qualificatif & 67 & & & & \\
\hline type de bilan / nom de bilan & 16 & & & & \\
\hline évaluateur & 58 & $4 \%$ & professionnel & 4 & $9 \%$ \\
\hline domaine / mécanisme & 255 & \multirow[b]{2}{*}{$21 \%$} & \multirow{2}{*}{$\begin{array}{l}\text { examen de capacité, fonction, } \\
\text { phénomène ou processus }\end{array}$} & \multirow{2}{*}{8} & \multirow{2}{*}{$19 \%$} \\
\hline domaine / pathologie & 47 & & & & \\
\hline
\end{tabular}

80

Au niveau de l'ensemble des sept pivots analysés en segments répétés, la comparaison des recensements par champ lexical et proportion de présence donne à voir la manière dont les champs lexicaux partagés structurent les pivots. Les résultats des différentes comparaisons que nous avons faites entre champs lexicaux sont représentés sous forme ensembliste.

81 La figure 3 montre un premier type de comparaison dans laquelle nous avons pris en compte les champs lexicaux présents pour au moins deux pivots et cela parmi les segments surreprésentés chez les orthophonistes et les non-orthophonistes.

Figure 3 : Champs lexicaux partagés par les segments répétés chez les orthophonistes et les nonorthophonistes

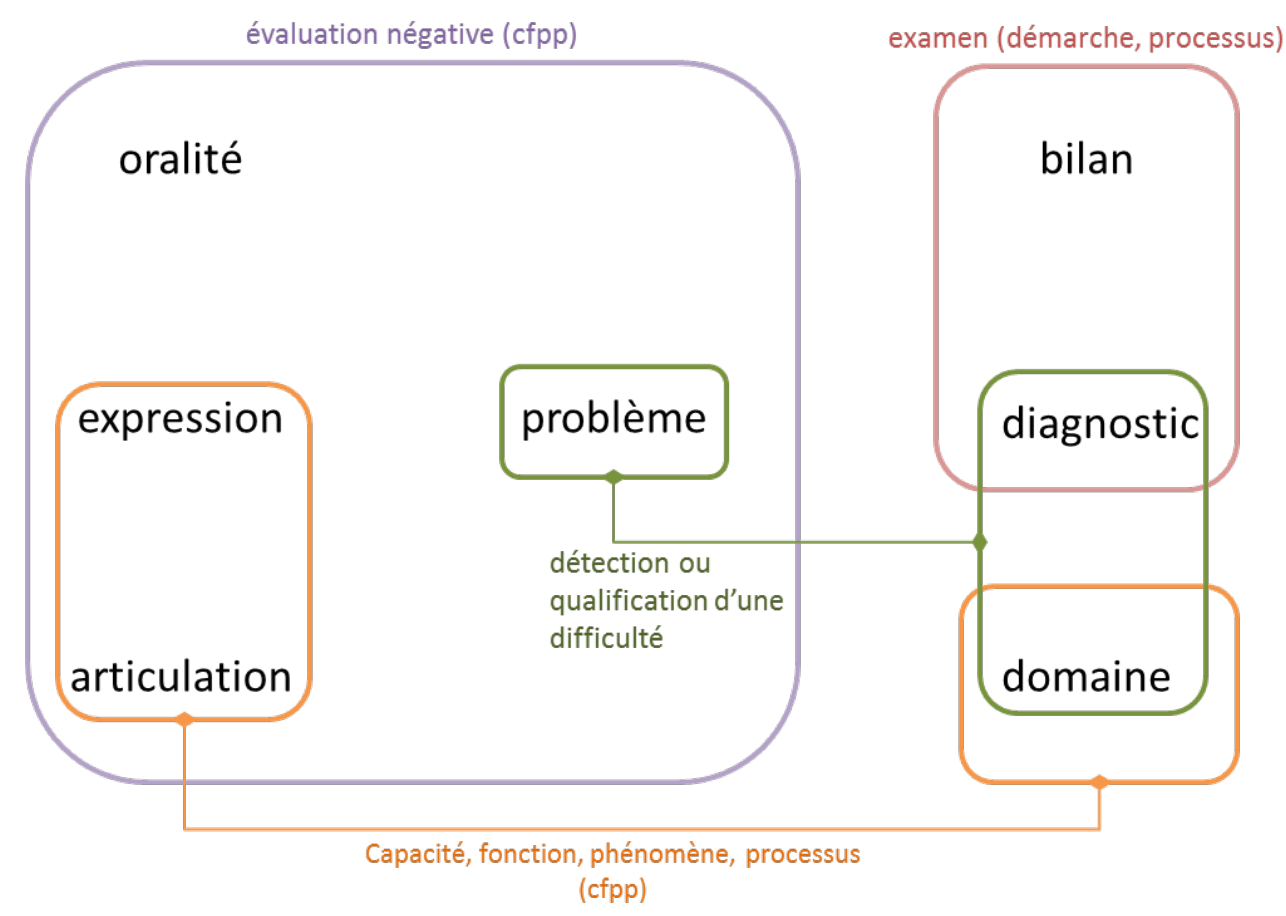

Ce premier type de comparaison fait apparaître quatre sous-groupes fondés sur le partage exclusif d'un ou deux champs lexicaux :

- le sous-groupe «bilan-diagnostic» partage les champs <examen (démarche)> et $<$ examen (processus)>;

- le sous-groupe "articulation-expression-oralité-problème» se structure autour du champ <évaluation négative d'une capacité, fonction, phénomène ou processus (cfpp)> ; 
- le sous-groupe «diagnostic-domaine-problème » est associé au champ <détection ou qualification d'une difficulté> ;

- le champ lexical <cfpp> établit un lien entre les deux plus grands sous-groupes: «articulation-expression-oralité-problème» d'une part et "diagnostic-domaineproblème » d'autre part.

Nous avons ensuite considéré les champs lexicaux partagés par les segments répétés surreprésentés uniquement dans le discours des orthophonistes. La structure qui en découle (figure 4), quoique plus difficile à interpréter de prime abord, montre une cohésion plus forte entre trois sous-groupes qui se dégagent.

Figure 4 : Champs lexicaux partagés dans le discours des orthophonistes

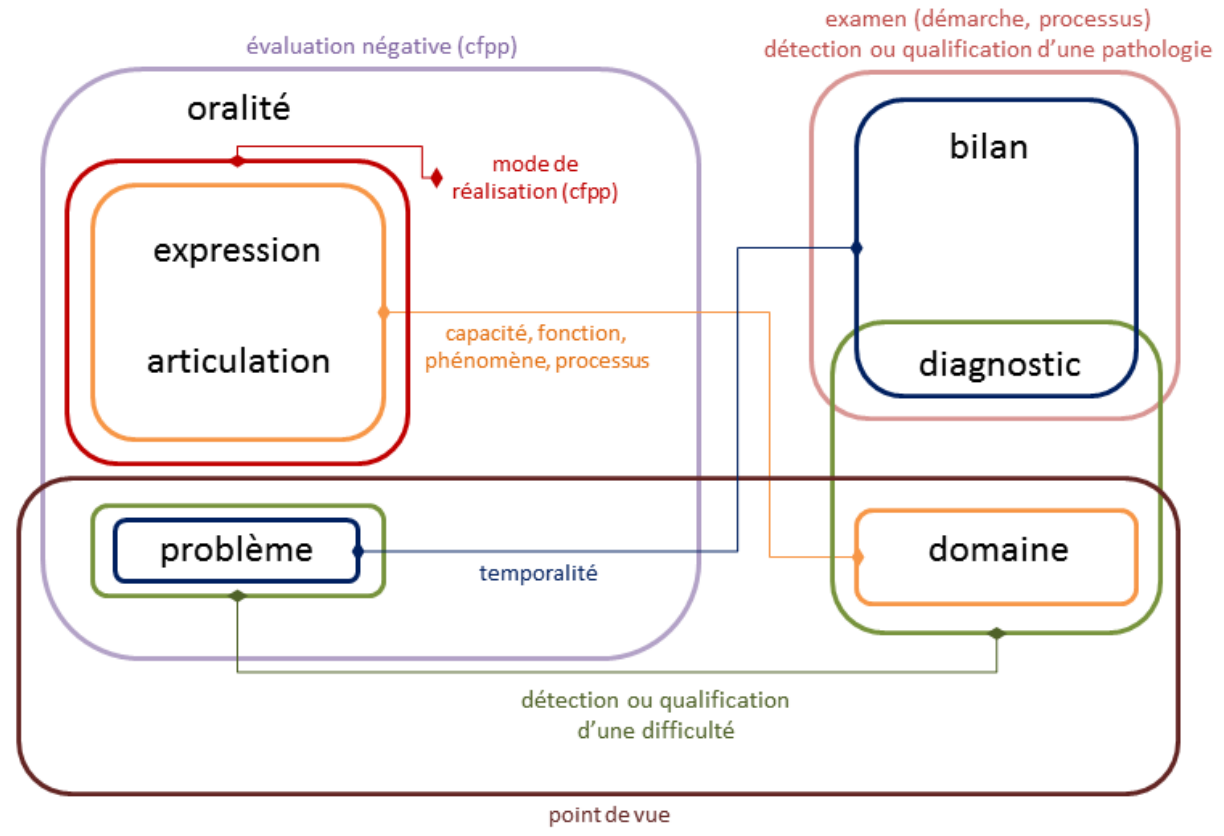


et établit un lien avec le sous-groupe « domaine-problème » via le pivot « problème ». Les champs <cfpp>, <détection ou qualification d'une difficulté>, <professionnel> et <temporalité> permettent de lier les trois sous-groupes principaux entre eux :

- comme précédemment, le champ <cfpp> établit un lien entre le sous-groupe «articulation-expression» et le sous-groupe «domaine-problème» via le pivot « domaine » et le champ < détection ou qualification d'une difficulté> établit un lien entre le sous-groupe «bilan-diagnostic » et le sous-groupe «domaine-problème » via le pivot « diagnostic »;

- le champ lexical <professionnel> établit un lien entre le sous-groupe "bilan-diagnostic » et le sous-groupe « domaine-problème » via le pivot « domaine »;

- le champ lexical <temporalité> enfin établit un lien entre le sous-groupe «bilandiagnostic » et le sous-groupe « domaine-problème » via le pivot " problème ».

Cette analyse en champs lexicaux partagés ne fournit pas une réponse définitive sur le statut central ou non central de chaque pivot. Cependant, elle permet de dégager des pistes de régularité à vérifier pour la structuration de la liste des sept pivots analysés.

Une hypothèse possible serait de considérer que les sous-groupes "articulationexpression » et « bilan-diagnostic » sont plutôt caractéristiques des orthophonistes étant donnée la charge sémantique des champs lexicaux qui assurent leur cohésion. Le premier sous-groupe pourrait faire référence aux objets (scientifiques) étudiés par les orthophonistes, notamment à cause du champ <mode de réalisation>. Le second sousgroupe pourrait faire référence à des méthodologies ou des outils d'étude grâce aux champs <examen (démarche et processus)> et <détection ou qualification d'une pathologie>.

89 Le sous-groupe « domaine-problème » pourrait être considéré comme périphérique à ces deux premiers sous-groupes. D'une part, le champ lexical qui assure sa cohésion, <point de vue>, est un champ que l'on pourrait s'attendre à trouver dans tout ou partie de la production scientifique. D'autre part, les champs lexicaux qui établissent des liens entre ce sous-groupe et les deux autres, à savoir les champs <détection ou qualification d222une difficulté>, <capacité, fonction, phénomène ou processus>, <évaluation négative d'une capacité ou d'une fonction, d'un phénomène ou d'un processus> et <temporalité> sont à la fois nombreux et très diversifiés sur le plan thématique.

90 Les pistes qui ont pu être dégagées en observant les champs lexicaux partagés par les pivots peuvent être approfondies en s'intéressant ensuite aux fréquences des champs lexicaux parmi les segments répétés autour des pivots. Nous avons réalisé cette étude en comparant les fréquences des champs lexicaux pour chaque sous-groupe identifié précédemment. Les fréquences sont calculées pour les segments surreprésentés dans le discours des orthophonistes d'une part, et dans celui des non-orthophonistes d'autre part. Ces fréquences correspondent à la proportion de segments répétés, auxquels est associé un champ lexical par rapport au nombre total de segments répétés du sousgroupe, pour les orthophonistes d'une part, et chez les non-orthophonistes d'autre part.

91 Lors de l'analyse des champs lexicaux partagés, le premier sous-groupe «articulationexpression» nous semblait faire référence aux objets scientifiques étudiés par les orthophonistes. L'examen des fréquences de champs lexicaux présents dans ce sousgroupe (figure 5) entre en résonance avec cette intuition sans pour autant la confirmer pleinement. 
Figure 5 : Champs lexicaux du sous-groupe articulation-expression pour les orthophonistes et les non-orthophonistes

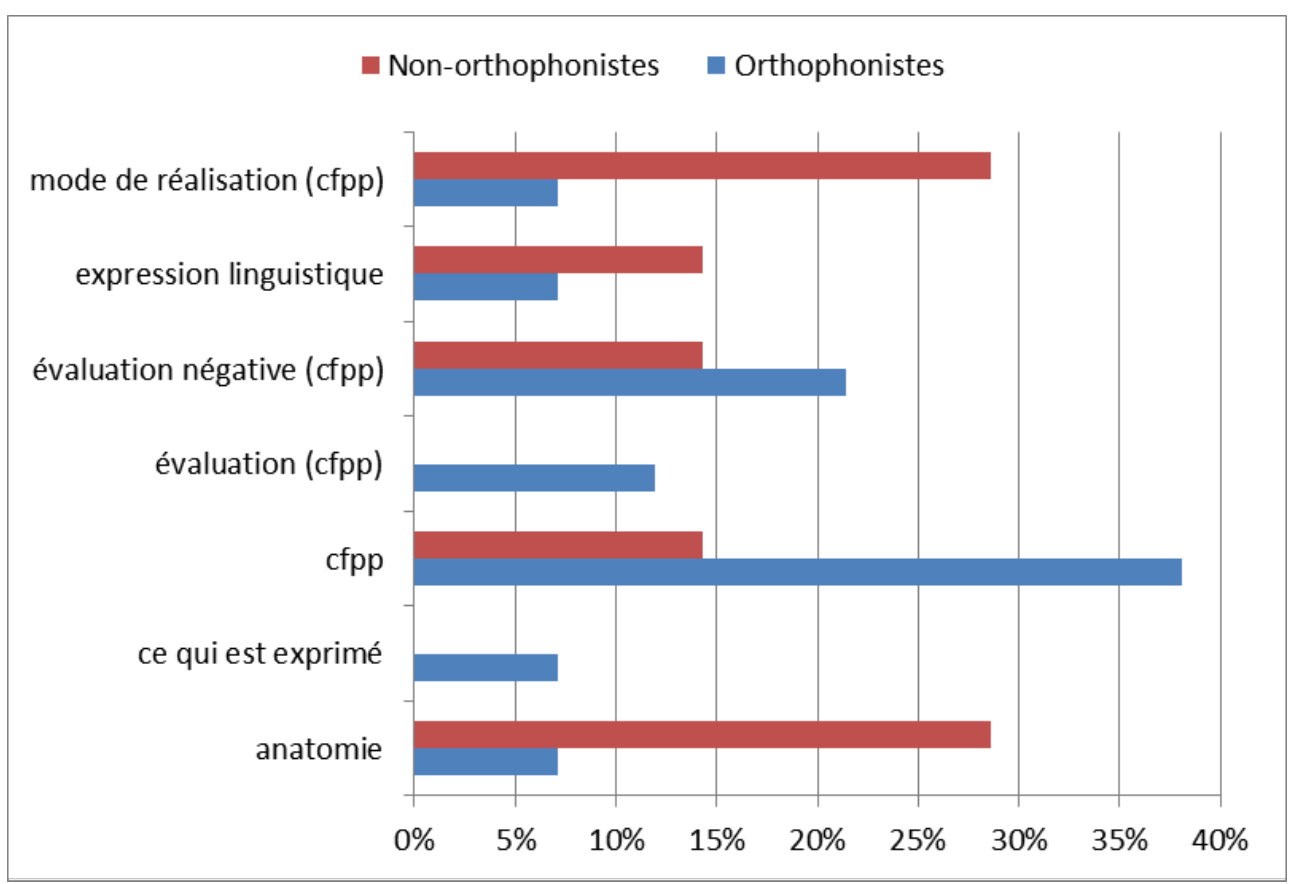

On remarque tout d'abord que le champ lexical assurant la cohésion du sous-groupe, le champ <mode de réalisation (cfpp)> (mode de réalisation d'une capacité, fonction, d'un phénomène ou d'un processus) est moins fréquent chez les orthophonistes. Ceci peut sembler surprenant, mais est contrebalancé par le fait que deux autres champs, <cfpp>, par exemple " articulation des consonnes », et <évaluation négative (cfpp)> (évaluation négative d'une capacité, fonction, d'un phénomène ou d'un processus), par exemple « des troubles de l'expression ", sont eux nettement plus fréquents chez les orthophonistes.

Ensuite, l'examen des fréquences des champs lexicaux dans le sous-groupe « articulationexpression » est révélateur des usages caractéristiques que les orthophonistes font des deux pivots. Le pivot « articulation » est ambigu entre plusieurs sens :

- deux sens relevant de l'orthophonie, représentés par le champ <cfpp> (capacité, fonction, phénomène, processus);

- un sens relevant de l'anatomie, représenté par le champ <anatomie>; un sens plus abstrait correspondant à l'articulation entre concepts ou entre deux démarches.

Le troisième sens n'est pas présent dans les segments répétés. Mais le sens relevant de l'anatomie, représenté par le champ <anatomie> avec par exemple "articulations temporo-mandibulaires", est nettement moins fréquent chez les orthophonistes. Autrement dit, l'analyse que nous avons réalisée des segments répétés autour du pivot «articulation» confirme que les orthophonistes utilisent ce terme davantage dans un sens relevant uniquement de l'orthophonie, et moins fréquemment dans un sens relevant de l'anatomie. Pour le pivot "expression", le même type d'observation peut être fait. Parmi les sens de ce pivot, le sens relevant de la linguistique, le type d'expression, par exemple "expression idiomatique», est nettement moins présent dans les segments répétés surreprésentés chez les orthophonistes que dans ceux qui sont surreprésentés chez les non-orthophonistes. À nouveau, cela montre l'usage moins fréquent que les orthophonistes font du sens linguistique du pivot «expression » par rapport à son sens 
orthophonique représenté ici par le champ <cfpp> (capacité, fonction, phénomène, processus).

Enfin, la dernière observation qui peut être faite de cet examen des fréquences à l'intérieur du sous-groupe «articulation-expression» concerne les champs qui n'apparaissent que dans les segments répétés surreprésentés chez les orthophonistes, à savoir les champs <ce qui est exprimé>, par exemple "expression de soi », <évaluation (cfpp)>), par exemple « capacités d'expression ». Ce dernier point invite à penser que les orthophonistes s'intéressent particulièrement à l'observation des <cfpp>, via l'analyse de ce que ces mécanismes ou capacités permettent de faire ou via l'examen de leur bon fonctionnement, mais surtout dans un axe individuel à chaque patient, et dans une globalité de fonctionnement.

Comme le montre la figure 4, dans le second sous-groupe, « bilan-diagnostic », les champs relevant de la notion d'examen vu sous l'angle d'un processus, sous l'angle de ce qu'on examine (<cfpp>) ou de l'objectif de l'examen sont les plus marqués chez les orthophonistes. Tous les autres champs présents dans ce sous-groupe sont, soit plus fréquents chez les non-orthophonistes (<détection et qualification d'une difficulté ou d'une pathologie>, <démarche d'examen>, <professionnel réalisant l'examen> ou <temporalité>), soit présents uniquement chez les non-orthophonistes (<évaluation d'une capacité ou fonction, d'un phénomène ou d'un processus>).

Figure 6 : Champs lexicaux dans le sous-groupe bilan-domaine pour les orthophonistes et les nonorthophonistes

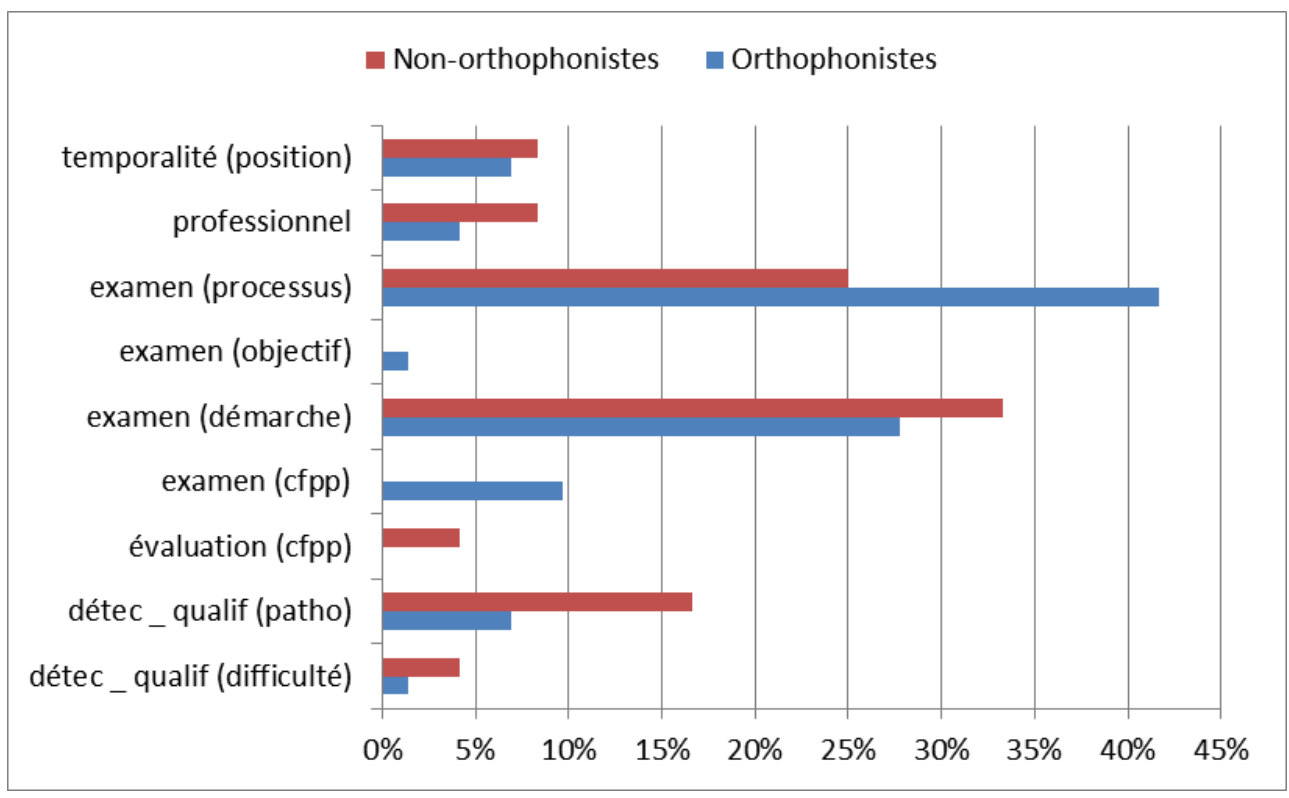

97 Il serait intéressant de comprendre pourquoi le champ <examen d'une capacité ou fonction, d'un phénomène ou d'un processus», par exemple "bilan langagier », est présent uniquement chez les orthophonistes et pourquoi le champ <évaluation d'une capacité ou fonction, d'un phénomène ou d'un processus», "bilan de langage » est présent uniquement chez les non-orthophonistes. La proximité entre les exemples associés à chacun des champs questionne l'association des étiquettes de champs lexicaux. Soit cette association est inutilement fine, soit il y a effectivement une préférence des orthophonistes pour la forme "bilan langagier » et une absence totale d'utilisation de la forme « bilan de langage ». 
98 En ce qui concerne le dernier sous-groupe, "domaine-problème », censé être plus périphérique par rapport au cœur des préoccupations des orthophonistes, l'observation des fréquences des champs lexicaux confirme cette hypothèse, mais à nouveau partiellement.

Figure 7 : Champs lexicaux du sous-groupe domaine-problème chez les orthophonistes et les nonorthophonistes

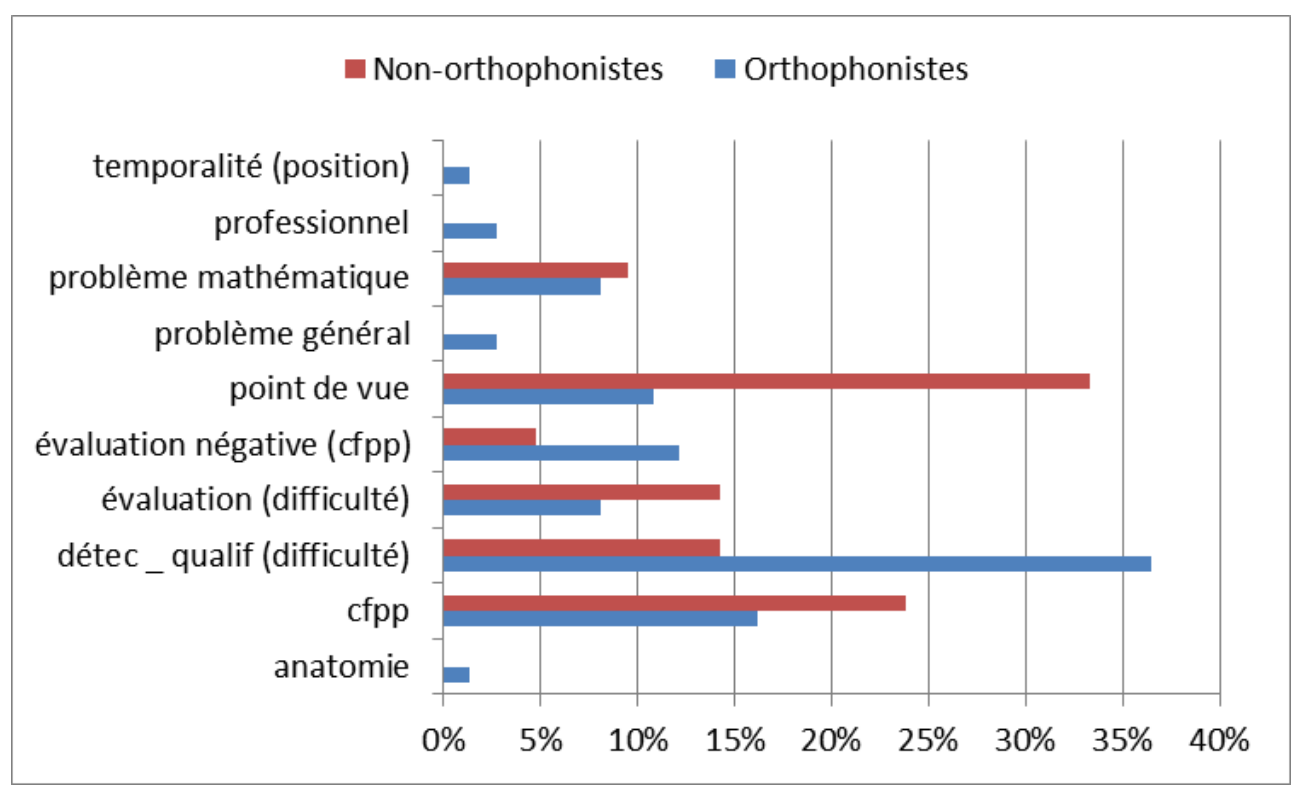

En effet, les champs les plus fréquents chez les non-orthophonistes relèvent soit d'un sens moins strictement orienté vers l'orthophonie, par exemple « résolution de problèmes » associé au champ <problème mathématique>, soit de sens courants dans l'écrit scientifique en général, par exemple « domaine de la recherche » associé au champ <point de vue> ou "problèmes majeurs" associé au champ <évaluation d'une difficultés. En revanche, le champ <capacité, fonction, phénomène, processus> apparaît étonnamment plus fréquemment chez les non-orthophonistes. Un examen détaillé des segments concernés montre que le pivot «domaine», avec "domaine du bégaiement» par exemple, joue systématiquement un rôle d'introducteur du mécanisme auquel il est fait référence et est donc transparent sémantiquement. Autrement dit, si une sélection sémantique des segments répétés avait été faite, de tels segments n'auraient pas été conservés.

Enfin, les champs lexicaux les plus fréquents chez les orthophonistes restent assez stables par rapport aux deux autres sous-groupes, mais à nouveau du fait du rôle d'introducteur que peuvent jouer les pivots «domaine » et "problème ». Concernant ces champs plus fréquents chez les orthophonistes, on notera tout de même le cas du champ <anatomie>, moins fréquent avec le sous-groupe "articulation-expression ", et seul présent dans ce sous-groupe « domaine-problème », avec l'unique segment " problèmes dentaires ».

\subsubsection{Les segments répétés vus comme des associations}

Dans un second temps, nous nous intéressons aux segments collectés en tant qu'associations récurrentes. Nous considérons alors que les segments rendent compte de la manière dont les orthophonistes combinent les pivots avec d'autres unités lexicales et 
nous cherchons à déterminer si ces combinaisons leur sont spécifiques ou si, à l'inverse, ce processus d'association est tout aussi fréquent chez les auteurs non orthophonistes de la revue.

Nous nous inspirons ici du «critère de cooccurrence différentielle» de Mel'čuk et al. [1995], sans en faire une application stricte. Nous partons de l'idée que si nous constatons que les orthophonistes ont une préférence marquée pour certains cooccurrents dans l'usage qu'ils font d'un pivot, nous avons un indice que ce pivot possède bel et bien un sens spécifique dans le domaine de l'orthophonie et qu'il est nécessaire de décrire ce sens. Pour chaque segment que nous avons identifié comme étant présent à la fois dans SC_ortho et dans le FrWac, nous reprenons les fréquences absolues collectées. Nous changeons en revanche la méthode de normalisation. La normalisation à 10 millions/kplaces utilisée précédemment nous permettait de nous faire une idée de la surreprésentation d'un segment donné par rapport à l'ensemble des segments de même taille possibles dans chaque (sous-)corpus. Ici nous souhaitons observer les segments par rapport à la fréquence de leur pivot dans chaque (sous-)corpus.

Le segment « expression orale », par exemple, est présent 66 fois dans SC_ortho, pour 882 occurrences d'« expression». Nous pouvons donc dire que le segment "expression orale » constitue $7,48 \%$ des occurrences de ce pivot dans le discours des orthophonistes. Dans le FrWac, la fréquence d'«expression orale " relative au nombre d'occurrences d'« expression » n'est que de $0,65 \%{ }^{11}$. Nous considérons donc qu'il s'agit d'une association récurrente surreprésentée chez les orthophonistes par rapport à la langue générale.

À l'issue de cette étape, nous constatons que le nombre d'associations surreprésentées dans la langue générale est supérieur au nombre de segments surreprésentés dans la langue générale que nous avons dénombré dans le tableau 16 de la partie 2.2.2. Ainsi, alors que nous n'avons constaté aucune surreprésentation impliquant « diagnostic » dans la langue générale avec la méthode de calcul précédente, " établir un diagnostic », " pour le diagnostic » et "sur le diagnostic » sont écartés lorsque nous les considérons comme des associations. Tous les pivots ne sont cependant pas également sensibles au changement de point de vue. Tandis que l'observation d' « expression » et d'« oralité » se révèle quasiment inchangée, l'ensemble des autres pivots est affecté. C'est pour " problème » que le taux d'associations écartées par rapport à la collecte initiale est le plus important, atteignant $10 \%$. Nous conservons toutefois un nombre intéressant de cooccurrents préférentiels aux orthophonistes pour chacun des pivots.

Tableau 20 : Associations surreprésentées chez les orthophonistes par rapport à la langue générale

\begin{tabular}{|c|c|c|c|c|}
\hline & $\begin{array}{l}\text { Effectif des associations } \\
\text { surreprésentées SC_ortho }\end{array}$ & $\begin{array}{l}\text { Effectif des associations } \\
\text { surreprésentées FrWac }\end{array}$ & $\begin{array}{l}\text { Effectif des associations } \\
\text { présentes uniquement dans } \\
\text { SC_ortho }\end{array}$ & Total \\
\hline articulation & 57 & 7 & 26 & 90 \\
\hline bilan & 122 & 5 & 53 & 180 \\
\hline diagnostic & 60 & 3 & 47 & 110 \\
\hline domaine & 111 & 13 & 53 & 177 \\
\hline \begin{tabular}{|l} 
expression \\
\end{tabular} & 86 & 1 & 13 & 100 \\
\hline \begin{tabular}{|l|} 
oralité \\
\end{tabular} & 15 & 0 & 37 & 52 \\
\hline problème & 169 & 23 & 37 & 229 \\
\hline
\end{tabular}

Nous concentrons ensuite notre attention sur les associations surreprésentées dans le discours des orthophonistes ou absentes du FrWac. Nous cherchons à déterminer si elles sont surreprésentées dans les articles écrits exclusivement par des orthophonistes par rapport aux autres. 
107 Le premier constat que nous faisons est que si les segments, observés en tant que tels et présents dans les deux parties du corpus ORTHOCORPUS, sont majoritairement surreprésentés chez les orthophonistes pour tous les pivots, ce n'est plus le cas pour «bilan» et "oralité » lorsque nous privilégions le point de vue de l'association. Cependant, le cas majoritaire reste celui d'associations apparaissant exclusivement dans les écrits des orthophonistes.

108 Le cas d'« oralité » est particulièrement frappant. Seuls les deux segments surreprésentés « oralité primaire » et «trouble de l'oralité » correspondent également à des associations surreprésentées. Il est d'ailleurs intéressant de noter que la forme plurielle «troubles de l'oralité » est pour sa part une association nettement surreprésentée chez les auteurs non orthophonistes, avec plus de $23 \%$ des emplois, contre un peu moins de $7 \%$ chez les orthophonistes.

Tableau 21 : Associations surreprésentées chez les orthophonistes par rapport à la revue RO

\begin{tabular}{|l|l|l|l|r|r|}
\cline { 2 - 6 } & \multicolumn{1}{c|}{$\begin{array}{l}\text { Effectif des associations } \\
\text { surreprésentées SC_ortho }\end{array}$} & $\begin{array}{l}\text { Effectif des associations } \\
\text { surreprésentées Sc_non-ortho }\end{array}$ & \multicolumn{2}{l|}{$\begin{array}{l}\text { Effectif des associations } \\
\text { présentes uniquement dans } \\
\text { SC_ortho }\end{array}$} & Total \\
\hline articulation & 16 & 9 & 58 & 83 \\
\hline bilan & 20 & 31 & 124 & 175 \\
\hline diagnostic & 40 & 20 & 47 & 107 \\
\hline domaine & 48 & 25 & 91 & 164 \\
\hline expression & 29 & 12 & 58 & 99 \\
\hline oralité & 2 & 14 & 36 & 52 \\
\hline problème & 67 & 23 & 116 & 206 \\
\hline
\end{tabular}

En dernier lieu, nous nous intéressons à la répartition des différents champs lexicaux à l'intérieur des sous-groupes qui ont émergé en 2.2.3. Nous souhaitons déterminer si les associations surreprésentées chez les deux catégories d'auteurs relèvent des mêmes champs que les segments répétés surreprésentés. 
Figure 8 : Comparaison entre segments et associations des fréquences de champs lexicaux dans trois sous-groupes
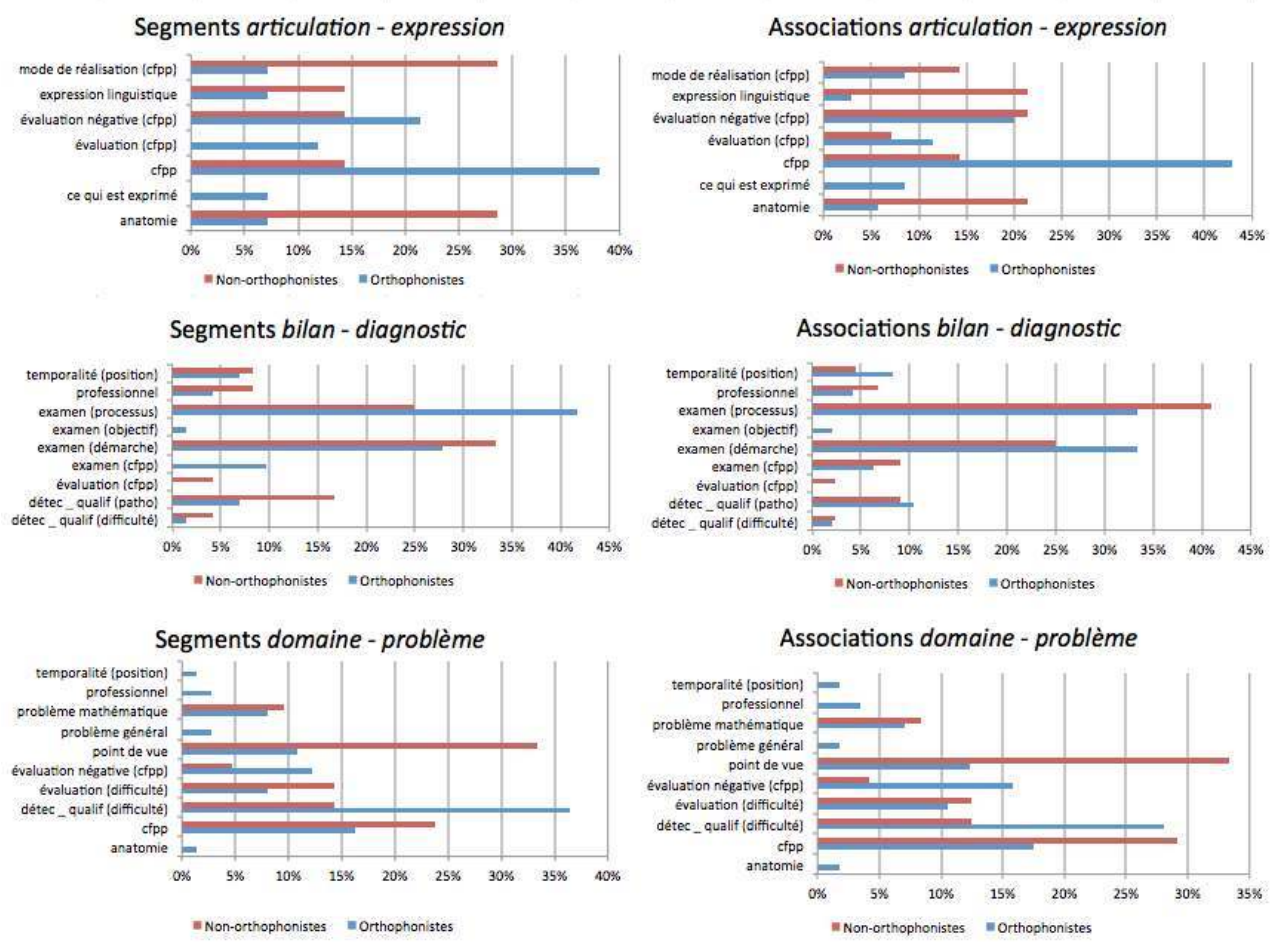

Dans le premier sous-groupe, constitué des pivots « articulation » et " expression », nous observons que les associations surreprésentées chez les orthophonistes se répartissent majoritairement dans les mêmes trois champs lexicaux que les segments répétés, le champ des capacités, fonctions, phénomènes et processus (<cfpp $>$ ) et ceux de leur évaluation (<évaluation (cfpp)> et <évaluation négative (cfpp)>). Le seul changement qui semble intéressant concerne le champ regroupant les formes dénotant des expressions linguistiques. Son poids diminue pour les orthophonistes, tout en augmentant chez les auteurs non-orthophonistes. Il en va de même pour le troisième sous-groupe, constitué des pivots « domaine » et « problème ». Aucun changement global n'est constaté, même si la préférence de certains champs par les orthophonistes ou les non-orthophonistes est nuancée. L'évaluation négative des capacités, fonctions, phénomènes ou processus (<évaluation négative (cfpp)>) est ainsi accentuée chez les orthophonistes, tandis que la proportion d'associations relatives à l'évaluation d'une difficulté (<évaluation (difficulté)>) est désormais très proche entre les deux populations.

Dans le second sous-groupe, constitué des pivots «bilan» et «diagnostic», les modifications apportées sont plus marquées. Alors que le champ dénotant l'examen orthophonique comme processus (<examen (processus)>) est seul en tête pour les segments répétés, sa surreprésentation diminue lorsque nous nous intéressons aux associations. S'il demeure fortement présent, il est désormais plus important pour les associations surreprésentées chez les non-orthophonistes. À l'inverse, les champs dénotant l'examen orthophonique comme démarche (<examen (démarche)>), ainsi que la détection et la qualification d'une pathologie (<détec_qualif (patho)>), apparaissent à présent comme davantage propres aux orthophonistes. 
112 Ces observations ne nous permettent pas directement de valider ou d'invalider le caractère central des UL étudiées. Cependant, elles jettent un regard nouveau sur les données disponibles et permettent de relativiser la surreprésentation de certains champs lexicaux dans les segments répétés autour de certains pivots pressentis comme termes centraux. Nous nous sommes cependant concentrées sur les cooccurrents communs aux orthophonistes et aux autres auteurs de la revue. Nous avons pu observer que, dans la majeure partie des cas, les cooccurrents lexicaux font l'objet d'une préférence d'emploi chez l'une ou l'autre de ces populations. Il serait désormais intéressant d'exploiter les segments répétés propres à chacun. Nous serions alors en mesure de compléter nos observations et de nous prononcer sur la nécessité d'intégrer des définitions propres aux orthophonistes pour les UL étudiées.

\section{Discussion et conclusion}

Dans cet article, nous avons relaté les étapes d'examen de 21 unités linguistiques utilisées en orthophonie, visant l'établissement de critères permettant de les évaluer comme étant centrales ou non dans le discours professionnel. L'objectif était de fournir une méthode de sélection comprenant une étape experte initiale dont les résultats pourraient être corroborés par des observations suivant des méthodologies d'analyse de corpus.

Dans un premier temps nous avons établi cette liste, qui a été soumise à un panel d'experts du domaine. Les critères retenus ont été la valeur notée de l'importance du terme en tant que révélateur des pratiques, et l'estimation de la spécificité de ce terme par rapport à l'usage dans d'autres professions. Enfin, la présence de dépendances semble être également assez signifiante. À ce stade, cinq UL semblaient unanimement être centrales ("articulation», "assourdissement», «jargon», "expression», " production »), quatre autres le sont moins franchement («bilan», « compréhension",

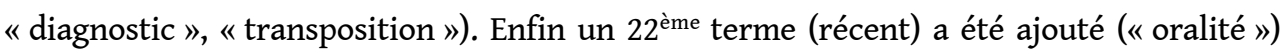
en raison de son actualité dans la profession.

115 Une deuxième étape semi-automatique a consisté en l'examen des concordances à droite et à gauche de chacune de ces UL, pour en dégager des tendances en termes de richesse et variétés des dépendances syntaxiques et sémantiques. De plus nous avons relevé les éventuelles entrées de ces termes dans le Dictionnaire d'orthophonie. Nous avons alors procédé à un examen approfondi de huit UL ayant un intérêt de par leur caractère censé central ou non central. Il s'agit d'« articulation ", «bilan ", " expression », " oralité », « diagnostic », « domaine », « problème » et « jargon ».

La troisième étape de traitement des données a consisté en l'extraction et l'analyse de segments répétés ayant pour pivot chacune des huit UL ayant été sélectionnées. Nous avons procédé à une analyse des champs lexicaux reliés aux segments répétés (sauf pour « jargon »). Ces étapes n'ont pas permis de répondre totalement à l'extraction de critères francs concernant le caractère central des termes, mais ont permis pour six UL de dégager trois sous-groupes: bilan-diagnostic, articulation-expression, et domaineproblème.

117 Enfin, nous nous sommes intéressées aux segments collectés en tant qu'associations ou combinaisons récurrentes. Ces résultats montrent l'intérêt de raisonner non seulement en termes quantitatifs, mais également qualitatifs, et notamment sur les champs lexicaux émanant de l'examen des segments répétés. 
118 La combinaison de ces étapes ne permet pas d'établir des conclusions franches concernant le caractère central de ces unités. Néanmoins, l'examen expert, manuel et semi-automatique des concordances et des segments répétés permet sans aucun doute d'obtenir un regard éclairé sur le comportement de chaque unité, d'effectuer dans certains cas des rapprochements (comme entre « diagnostic » et «bilan » par exemple). Il semble que comme pour tout objet de recherche, les résultats de l'examen proposé dépendent de la façon dont on l'observe.

Pour conclure sur cette série d'observations, nous pensons que les unités lexicales que nous pouvons établir comme centraux sont "bilan », "diagnostic ", " articulation », et « expression ». Ces unités sont largement utilisées pour décrire les capacités des patients, pour évoquer l'évaluation de ces capacités (en tant que démarche utile). Concernant les caractéristiques de ces UL, et de façon assez attendue, les indices nombreux relevés dans ces étapes ne concordent que dans certains cas pour établir que l'UL est caractéristique du discours des orthophonistes. Ces indices sont le regard expert, la densité et la variété des concordances et la surreprésentation des champs lexicaux associés à certains segments. La détermination de ce qui est important et révélateur des pratiques, ou spécifique aux orthophonistes, relève d'une discussion théorique extrêmement intéressante sur le plan sémantique et morphologique, et complète parfaitement le travail engagé sur cette thématique depuis les premiers essais lexicographiques publiés à la fin du XXe siècle. Un des intérêts de ce travail aura été de sélectionner des termes méritant de bénéficier d'une modification ou d'une création de définition dans la nouvelle version à venir du Dictionnaire d'orthophonie. Par ailleurs, ce travail fournit une photographie intéressante de l'usage de ces UL dans un corpus spécifique d'articles scientifiques en orthophonie et contribue à une meilleure connaissance du domaine.

Au-delà de l'examen terminologique sur les termes diagnostiques (les noms des pathologies du langage, de la communication et des fonctions orofaciales dont s'occupent les orthophonistes), l'examen de termes dits centraux permet d'enrichir la vision de la langue de spécialité. En effet les procédures adoptées qui ont été décrites ici montrent combien la perception de l'importance et de la spécificité d'une unité lexicale peut être complexe. En ce sens, ce travail permet d'apporter des éléments concernant l'exploration des possibilités et des limites de l'utilisation complémentaire de diverses méthodes d'analyse de corpus pour analyser la portée sémantique spécifique d'un terme dans un domaine de spécialité. L'interdisciplinarité et le va-et-vient entre des modalités d'analyse à la fois qualitatives et quantitatives, et entre des considérations disciplinaires et linguistiques, nous semble extrêmement judicieux.

Les limites de ce travail ne sont pas négligeables. En premier lieu le panel d'experts n'était ni suffisant ni totalement représentatif. De plus il semble à la lumière de nos analyses qu'une annotation complète des concordances en classes sémantiques et syntaxiques, congruente sur les plans à la fois disciplinaire et linguistique, est bien indispensable mais qu'elle ne sera pas toujours réalisable en pratique dans une perspective lexicographique.

122 Enfin, il semble utile de compléter l'analyse réalisée à partir des segments répétés partagés par le discours des orthophonistes et celui des non-orthophonistes dans la revue Rééducation Orthophonique, et ce dans deux directions. Il s'agit de procéder d'une part à une analyse détaillée des segments répétés employés uniquement par les auteurs orthophonistes, et d'autre part au recueil et à l'analyse des segments répétés employés uniquement par les auteurs non orthophonistes. 
Copie d'écran du tableur envoyé aux orthophonistes, avec la question : « D’après vous, le mot suivant » :

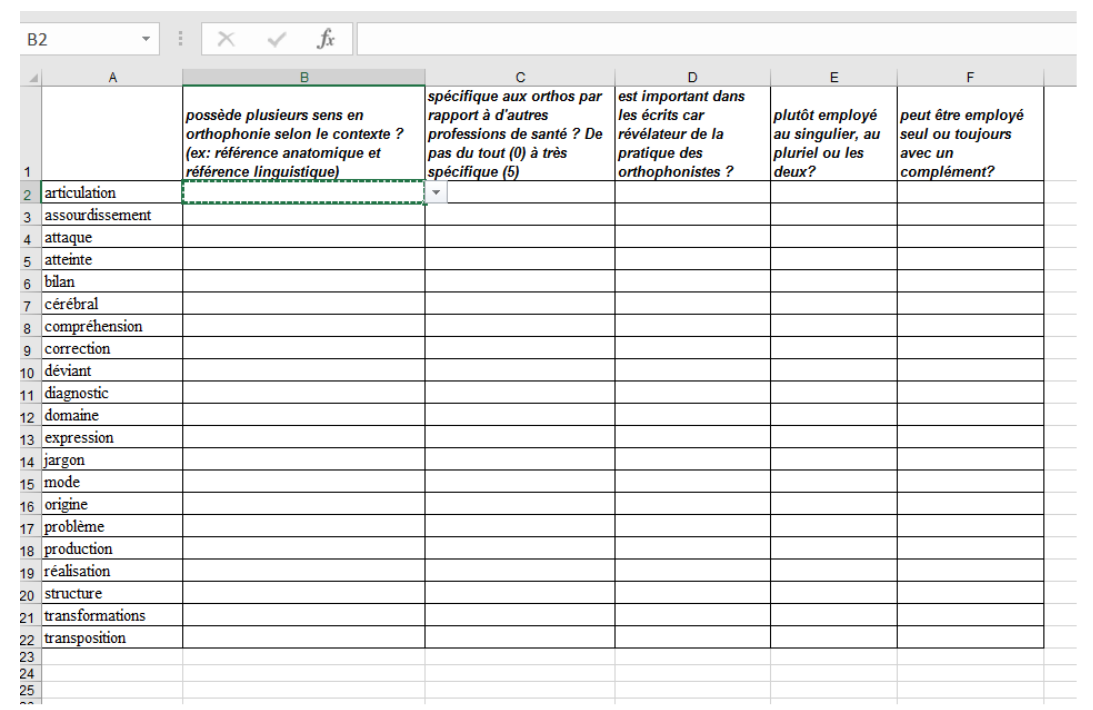

\section{BIBLIOGRAPHIE}

BARONI Marco et al., 2009, “The WaCky wide web: a collection of very large linguistically processed web-crawled corpora”, Language Resources and Evaluation 43 (3), 209-226, http://nl.ijs.si/noske/ wacs.cgi/first_form

BRIN-HENRY Frédérique, 2014, « Using corpus-based analyses in specialised paramedical French », Revue Française de Linguistique Appliquée, 19 (1), Langues de spécialité : problèmes et méthodes, 103-115. BRIN-HENRY Frédérique \& KNITTEL Marie-Laurence, 2015, « L'usage des termes difficulté(s) et trouble (s) dans un corpus de comptes rendus de bilans orthophoniques ", Communication présentée lors du colloque « Cures de langage(s) », Arras, 10-11 décembre 2015.

BRIN-HENRY Frédérique \& KNITTEL Marie-Laurence, 2016, « Etude lexicosémantique du nom

difficulté(s) dans les comptes rendus de bilan orthophonique : apports structuraux et conceptuels », LIDIL 53, 19-41.

BRIN-HENRY Frédérique, 2017, « Oralité(s) : origine terminologique et évolution conceptuelle en orthophonie », Rééducation Orthophonique 271, Isbergues : Ortho Edition, 435-449.

BRIN-HENRY Frédérique, COURRIER Catherine, LEDERLÉ Emmanuelle \& MASY Véronique, 2011 [1997], Dictionnaire d'orthophonie, Isbergues : Ortho-Edition.

CABRÉ Maria Teresa, 2012, « Disciplinarisation de la terminologie : contribution de la linguistique », Dossiers d'HEL-SHESL, 1-10.

CHARAUdeAu Patrick \& MAINGUENEAu Dominique, 2002, Dictionnaire d'Analyse du Discours, Paris : Éditions du Seuil. 
GADER Nabil, LUX-POGODALLA Veronika \& POLGUÈre Alain, 2012, "Hand-Crafting a Lexical Network with a Knowledge-Based Graph Editor", Proceedings of the Third Workshop on Cognitive Aspects of the Lexicon (CogALex III), 109-125.

HEIDEN Serge, MAGUÉ, Jean-Pierre \& PINCEMIN, Bénédicte, 2010, « TXM : Une plateforme logicielle open-source pour la textométrie - conception et développement ", in BoLASCo Sergio, CHIARI Isabella \& GIULIANo Luca (eds.), Proc. of $10^{\text {th }}$ International Conference on the Statistical Analysis of Textual Data, JADT 2010, vol. 2, 1021-1032, Rome, Italie : Edizioni Universitarie di Lettere Economia Diritto, http://textometrie.ens-lyon.fr/

LINo Maria Teresa, 2006, «Contextes et néologie terminologique dans le domaine médical », Mots, termes et contextes, 509-514.

L'Hoмme Marie-Claude, 2005, « Sur la notion de "terme" ", Meta : journal des traducteurs/Meta: Translators' Journal 50 (4), 1112-1132.

LUX-POGODALLA Véronique \& POLGUÈRE Alain, 2011, "Construction of a French Lexical Network: Methodological Issues", First International Workshop on Lexical Resources, WoLeR 2011, Ljubljana, Slovenia, 54-61.

MAYAFFRE Damon \& VIPReY Jean-Marie, 2012, « Présentation », Corpus 11, http:// corpus.revues.org/ 2200

MEL'Čuk Igor et al., 1995, Introduction à la lexicologie explicative et combinatoire, Paris/Louvain-laNeuve : Duculot.

NEE Emilie, SITRI Frédérique \& VENIAR, Marie, 2014, « Pour une approche des routines discursives dans les écrits professionnels », in Neveu F., Blumenthal P., Hriba L., Gerstenberg A., M

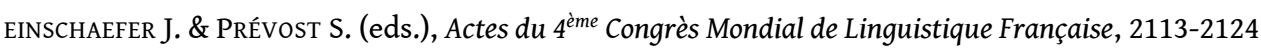

SALEM André, 1987, Pratique des segments répétés. Essai de statistique textuelle, Paris : Klincksieck, Publications de l'INALF, coll. « Saint-Cloud».

SCHMID Helmut, 1994, "Probabilistic part-of-speech tagging using decision trees", Proceedings of International Conference on New Methods in Language Processing, Manchester, Royaume-Uni, 1-9.

\section{NOTES}

1. Projet soutenu par la région Lorraine, l'ATILF, le $\mathrm{CH}$ de Bar-le-Duc et la Fédération Nationale des Orthophonistes.

2. Chaque expert peut être comptabilisé dans plusieurs catégories.

3. http://www.fno.fr/27eme-congres-scientifique-international-de-la-fno-du-19-au-21octobre-2017-a-strasbourg/programme-du-congres-de-strasbourg/

4. Revue éditée depuis 1997 par Ortho Edition, disponible à : https://www.orthoedition.com/ revues/abonnement-a-reeducation-orthophonique-174.html

5. Analyse et traitement informatique de la langue française - UMR 7118 (ATILF) (2018), OrthoCorpus [Corpus], ORTOLANG (Open Resources and TOols for LANGuage) : www.ortolang.fr

6. Le corpus a été interrogé grâce aux fonctionnalités de la plateforme de textométrie TXM, disponible à : http://textometrie.ens-lyon.fr/

7. http://www.larousse.fr/dictionnaires/francais

8. Pour des raisons de lisibilité, ce sous-corpus sera par la suite appelé SC_ortho. 
9. Le corpus FrWac est le résultat d'une extraction de pages Web en français au cours de l'année 2008. Ce corpus contient 1,6 milliards de tokens et est mis à disposition de la communauté sous forme brute ou sous forme étiquetée en morphosyntaxe à l'aide du logiciel TreeTagger [Schmidt 1994].

10. Ce second sous-corpus sera par la suite appelé SC_non-ortho.

11. Nous comptabilisons 481.413 occurrences d'« expression» et 3.147 occurrences $\mathrm{d}^{\prime}$ ' expression orale ».

\section{RÉSUMÉS}

Nous nous intéressons depuis une dizaine d'années au discours professionnel et scientifique des orthophonistes. Un de nos projets actuels porte sur l'identification de ce que nous appellerons désormais des termes centraux appartenant au français de l'orthophonie. Il s'agit d'unités lexicales (UL) dont l'usage est particulier dans le discours des orthophonistes et qui sont considérées comme révélatrices des pratiques de ces professionnels et chercheurs. L'objectif est la mise au point d'une méthode reproductible de sélection de termes révélateurs des pratiques de cette profession de santé, afin de nourrir la réflexion terminologique du domaine.

Une liste de 21 UL a été établie par l'une des auteures (orthophoniste) et soumise à une validation par un panel d'autres experts orthophonistes. En parallèle, nous avons développé une méthodologie issue de la linguistique de corpus pour repérer et caractériser l'usage des termes centraux dans le discours des orthophonistes. Cette méthodologie se fonde sur des analyses habituelles de concordances, de fréquences et applique une forme d'étude des segments répétés. Elle s'appuie sur le corpus du projet OrthoCorpus, constitué de 850 articles scientifiques provenant de la revue Rééducation Orthophonique sur une période allant de 1997 à 2014.

Le présent article expose les étapes complémentaires que nous avons mises en œuvre, ainsi que les résultats pour huit des UL examinées en détail. Les résultats sont concordants pour quatre de ces UL. Nous présentons ensuite les conséquences de ces résultats pour la suite de nos analyses.

We have been studying the professional and scientific discourse of speech and language therapists (SLTs) for more than ten years. One of our projects currently focuses on the identification of what we will refer to as central terms among French speech therapists. These lexical units are used in a particular way by SLTs. They may be considered as very typical in that they tend to contribute to the characterisation of SLT practice. The aim of our study is first to establish a reproducible method for selecting terms that are useful for the characterisation of SLT practice, and then to contribute to the definition of its terminology.

A list of 21 lexical units was drawn up by one of the authors (who is also a SLT), and submitted to a panel of other SLT experts. Concurrently, we developed a corpus-based method in order to identify and describe the use of central terms in SLT discourse. Usual analyses on concordances were conducted, as well as frequency measures and examination of regular expressions. We used a corpus built during the OrthoCorpus project. The corpus contains 850 scientific articles which were published between 1997 and 2014 in the French SLT journal entitled Rééducation Orthophonique.

In this article, we describe each additional step we took and the results we found for eight of the examined lexical units. Our results are consistent for four units. We then conclude on the consequences these results may have on the future of our work. 
INDEX

Keywords : corpus linguistics, speech and language therapy, specialised language, central terms Mots-clés : linguistique de corpus, orthophonie, langue de spécialité, termes centraux

\section{AUTEURS}

\section{FRÉDÉRIQUE BRIN-HENRY}

Centre Hospitalier de Bar-le-Duc 55 et laboratoire ATILF-UMR 7118 CNRS / Université de Lorraine frederique.henry@atilf.fr

\section{ÉVELYNE JACQUEY}

Laboratoire ATILF-UMR 7118 CNRS / Université de Lorraine

Evelyne.Jacquey@atilf.fr

\section{SANDRINE OLLINGER}

Laboratoire ATILF-UMR 7118 CNRS / Université de Lorraine

Sandrine.ollinger@atilf.fr 